
порядка, временные причинные процессы

Для цитирования: Вирбицкайте И.Б., Зубарев А.Ю. Временные причинно-упорядоченные процессы временных сетей Петри со «слабой» семантикой. Труды ИСП РАН, том 32, вып. 4, 2020 г., стр. 261-284 (на английском языке). DOI: 10.15514/ISPRAS-2020-32(4)-19

\title{
Time Causal Processes in Time Petri Nets with Weak Semantics
}

1,2I.B. Virbitskaite, ORCID:0000-0002-4475-3480<virb@iis.nsk.su>

${ }^{1}$ A.Yu. Zubarev, ORCID: 0000-0002-3499-3253 <auzubarev@gmail.com>

${ }^{1}$ A.P. Ershov Institute of Informatics Systems, SB RAS

6, Acad. Lavrentiev avenue, 630090, Novosibirsk, Russia

$$
{ }^{2} \text { Novosibirsk State University, }
$$

2, Pirogova st., Novosibirsk, 630090, Russia

Abstract. In this paper, we present a method for state space reduction of dense-time Petri nets (TPNs) - an extension of Petri nets by adding a time interval to every transition for its firing. The time elapsing and memory operating policis de cont discrete, in general, and, therefore, the analysis of its behavior is rather complicated. To cope with the problem, we elaborate a state space discretization technique and develop a partial order semantics for TPNs equipped
with weak time elapsing and intermediate memory policies.

Keywords: dense-time Petri nets; weak time elapsing semantics; intermediate memory policy; state space discretization; partial order semantics; time causal processes

For citation: Virbitskaite I.B., Zubarev A.Yu. Time Causal Processes in Time Petri Nets with Weak Semantics. Trudy ISP RAN/Proc. ISP RAS, vol. 32, issue 4, 2020, pp. 261-284. DOI: 10.15514/ISPRAS-2020-32(4)-19

\section{Временные причинно-упорядоченные процессы временных} сетей Петри со «слабой» семантикой

1,2 И.Б. Вирбиикайте, ORCID: 0000-0002-4475-3480<virb@iis.nsk.su> ${ }^{1}$ A.Ю. 3убарев, ORCID:0000-0002-3499-3253 <auzubarev@gmail.com>

${ }^{1}$ Институт систем информатики им. А.П. Ершова СО РАН, 630090, Новосибирск, проспект Лаврентьева, 6 ${ }^{2}$ Новосибирский государственный университет 630090, Новосибирск, ул. Пирогова, 2

Abstract. В данной статье предлагается метод редукции пространства состояний непрерывновременных сетей Петри (НВСП) - расширения сетей Петри, где каждому переходу ставится в соответствие временной интервал его срабатывания. Техники контроля времени и памяти определяют различные семантики для НВСП, которые влияют на разрешимость многих стандартных проблем анализа поведения НВСП. В общем случае, пространство состояний НВСП бесконечно и несчетно, и, следовательно, анализ их поведения довольно сложен. С целью разрешения данной проблемы выполняется дискретизация пространства состояний и определяется семантика частичного порядка для НВСП со «слабой» техникой продвижения времени (продвижение времени неограничено) и «промежуточной» техникой контроля памяти (с учетом промежуточных разметок при срабатывании сетевых переходов)

\section{Introduction}

Dense-Time Petri Nets (TPNs) are now a well-established model to describe and study safety-critical systems that often require verification of real time (quantitative) characteristics, in addition to functional (qualitative) properties. In the TPN, each transition is associated with a time interval. With that, each transition is assumed to have its own local clock. A state of the TPN contains a current marking and readings of the local clocks of enabled transitions (i.e. transitions whose all input places have enough tokens at the marking). A transition can fire from a state only if the transition is enabled at the corresponding marking and its clock reaches a moment in time that is within the interval associated. So, the firing of an enabled transition can be suspended for a certain time. Along with that, the firing itself takes no time. State changes are divided in two types: either time elapses, i.e. the clocks of enabled transitions go forward, or a transition fires, i.e. a curren marking is changed to a new one (in which tokens are consumed from the input places and tokens are produced to the output places of the transition that fires) and the clocks of the transitions that become enabled at the new marking (newly enabled transitions) are reset to zero.

There are two policies of time elapsing in TPNs, which define strong and weak semantics. In the former semantics, time elapsing cannot exceed the upper bounds of enabled transitions and, therefore, an enabled transition must fire no later than the upper bound of its time interval is reached. On the contrary, any time elapsing is allowed in the latter semantics and, therefore, enabled transitions are not forced to fire. In [1], the authors have proven that the two semantics are incomparable w.r.t. timed weak bisimulation.

Memory policies in TPNs determine when the local clocks of enabled transitions are reset. Intermediate and atomic memory policies are put forward in the literature. The former treats intermediary marking, i.e. the marking after consumption of tokens from the input places and before production of tokens to the output places of a transition $t$ that fires. A transition $t^{\prime}$ is regarded as newly enabled and its clock is reset to zero after the firing of $t$ whenever $t^{\prime}$ is disabled at the intermediary marking and becomes enabled at the new marking, i.e. after production of tokens to the output places of $t$. Instead, the latter policy considers a firing as one-step. The clock of $t^{\prime}$ is reset to zero only if it is disabled at the marking before $t$ fires and becomes enabled at the new marking after $t$ fires. The memory policies were studied in [2] for strong semantic and in [3] for weak semantics. It was shown that the marking reachability/coverability and boundedness problems are undecidable for time Petri nets with strong semantics and any memory policy, whereas the problems are decidable in the case of TPNs with weak intermediate semantics but not with weak atomic semantics.

The state space of the TPN is infinite and non-discrete, in general, that increases the difficulty of the model analysis. In the work [4], a transformation to the behavior with only integer time elapsings has been suggested for TPNs with strong semantics, while the discretization of the state space for weak semantics has hitherto not be treated in the literature, to the best of our knowledge.

The classical interleaving behavior of the TPN is described by runs - sequences of changes in states by time elapsings or transition firings. Interleaving semantics allows for analyzing some safety and liveness properties of systems. However, using partial order semantics seems preferable because it captures in a natural way «true concurrency». Partial order semantics of Petri nets is most often represented by means of the so-called causal net processes, which include events and conditions related by causal dependence and concurrency. This information can be useful for forma 
verification of the system behavior or for reducing the number of analyzed system states, without taking into account all interleaving sequences. Partial order semantics is put forward for safe TPNs with strong and clocks-on-transitions semantics in [5]. The presented in [6] approach to construct a partial order and non-deterministic representation of the behavior of safe TPNs with strong and clocks-on-tokens semantics consists in transforming time characteristics into net structure, i.e. representing them by additional places, transitions, and arcs. This allows for removing the restrictions of diverging time and of finite upper time bounds for transitions. In [7], the authors inspect free choice TPNs (i.e. net transitions sharing an input place do have exactly the same input places), develop and compare partial order representations of runs, based on various clocks-ontokens semantics.

In this paper, we deal with dense-time Petri nets with weak and intermediate policies. Our intention here is twofold. First, we develop a discrete representation of the interleaving behavior (runs) of the TPN by transforming its runs with real-number time elapsings to parametric sequences with time variables that are then assigned natural-number values. Second, partial order clocks-on-tokens semantics in terms of time causal processes of the TPN, by converting time elapsings into net structure, is elaborated. Also, for the TPN, a bijective mapping between its runs and computations (called linearizations) of its time causal processes is constructed, in order to demonstrate the correctness of the partial order semantics w.r.t. interleaving one. Partial order semantics allow for taking into account the processes' timing behavior in addition to their degrees of relative concurrency.

The paper is organized as follows. In Section 2, we consider some definitions for TPNs and their interleaving semantics in terms of runs - sequences of changes in states by time elapsings and transition firings. In the following section, it is established that the discretization of TPN's state space is possible by demonstrating that in the TPN for any run with transition firings and realnumber time elapsings there exists a run having the same transition firings and only natural-number (even unit) time elapsings. In Section 4, we introduce and examine properties of a casual net, its linearization, and a time causal process of the TPN, consisting of a casual net and its homomorphism into the TPN. In the next section, a bijective mapping from a set of linearizations of causal nets of time processes of the TPN to its set of runs is developed and studied. Section 6 concludes the paper.

\section{Time Petri Nets}

In this section, some terminology concerning the model of Petri nets with timing constraints (time intervals on the firings of transitions) are defined. We start with recalling the definitions of the structure and behavior of Petri nets.

The Petri net (PN) consists of two different sets of elements - places and transitions; a flow relation representing arrows between the elements; an initial marking - a subset of places initially containing tokens; and a labeling function mapping each transition to an action from the alphabet Act of actions. A state of the PN is called a marking - a subset of places that receive tokens when the net functions. A transition is enabled at a marking if the input places of the transition contain tokens. The firing of a transition enabled at a marking results in the new marking in which tokens are consumed from the input places and tokens are produced to the output places of the transition. A sequence of changes in markings is called a run of the PN.

Definition 1. A (labeled over Act) Petri net (PN) is a tuple $\mathcal{N}=\left(P, T, F, M_{0}, L\right)$, where $P$ is a finite set of places and $T$ is a finite set of transitions such that $P \cap T=\varnothing$ and $P \cup T \neq \varnothing$; $F \subseteq(P \times T) \cup(T \times P)$ is a flow relation; $\varnothing \neq M_{0} \subseteq P$ is an initial marking; $L: T \rightarrow$ Act is a labeling function. For $x \in P \cup T$, let $\bullet x=\{y \mid(y, x) \in F\}$ and $x \bullet=\{y \mid(x, y) \in F\}$ be the preset and postset of $x$, respectively. For $X \subseteq P \cup T$, define $\bullet X=\cup_{x \in X} \bullet x$ and $X \bullet=$ $\mathrm{U}_{x \in X} x \bullet$
A marking $M$ of a Petri net $\mathcal{N}$ is any subset of $P$. A transition $t \in T$ is enabled at a marking $M$ if $\bullet \subseteq M$. Let $E n(M)$ be the set of transitions enabled at $M$.

The firing of a transition $t$ enabled at a marking $M$ leads to the new marking $M^{\prime}$ (denoted $M$ $\left.\stackrel{t}{\rightarrow} M^{\prime}\right) \quad$ iff $\quad M^{\prime}=(M \backslash \bullet t) \cup t \bullet$. We write $\quad M \stackrel{\vartheta}{\rightarrow} M^{\prime} \quad$ iff $\quad \vartheta=t_{1} \ldots t_{k} \quad$ and $\quad M=$ $M^{0} \stackrel{t_{1}}{\rightarrow} M^{1} \ldots M^{k-1} \stackrel{t_{k}}{\rightarrow} M^{k}=M^{\prime}(k \geq 0)$. In this case, $\vartheta$ is a run of $\mathcal{N}$ from $M$ (to $\left.M^{\prime}\right)$, and $M^{\prime}$ is a reachable marking of $\mathcal{N}$ from $M$. Let $\mathcal{R} \mathcal{M}(\mathcal{N})$ be the set of all reachable markings of $\mathcal{N}$ from $M_{0}$.

The time Petri net (TPN) consists of an underlying PN and a static timing function mapping each transition to a time interval with non-negative rational boundaries. With that, each transition is assumed to have its own local clock. A marking alone is not enough to describe a state of the TPN, so a dynamic timing function is added to indicate the clock values of the transition enabled at current marking. In fact, the clocks of enabled transitions show the times passed since then as the transitions become enabled. The initial state consists of the initial marking and the dynamic timing function with zero clock values for all enabled transitions. When the TPN is running, there are two ways to change states: either by time elapsings or by transition firings. Following the approach of [3], we consider TPNs with weak semantics. This means that any time elapsing is allowed, i.e. any time can be added to the clock values of enabled transitions. A transition can fire from a current state only if the transition is enabled at the current marking and its clock value belongs to its time interval. The firing of a transition that can fire from a state results in a new state, i.e. a new marking and new dynamic timing function with the clock values reset to zero for the newly enabled transitions and with the old clock values for the transitions which continue to be enabled. We deal with TPNs with intermediate memory policy, i.e. the predicate $\uparrow \operatorname{enabled}\left(t^{\prime}, M, t\right)$ determining a newly enabled transition $t^{\prime}$ after the firing of a transition $t$ at a marking $M$ has a true value if and only if $t^{\prime}$ is disabled at intermediary marking (i.e. the marking between consumption and production of tokens by the firing of $t$ ) and becomes enabled at the new marking (i.e. the marking after production of tokens by the firing of $t$ ). A sequence of changes in states is called a run of the TPN. The runs from the initial state represent interleaving semantics of the TPN

Definition 2. A (labeled over Act) time Petri net (TPN) is a pair $\mathcal{T N}=(\mathcal{N}, D)$, where $\mathcal{N}=$ $\left(P, T, F, M_{0}, L\right)$ is the underlying Petri net and $D: T \rightarrow \mathbb{Q}_{\geq 0} \times\left(\mathbb{Q}_{\geq 0} \cup\{\infty\}\right)$ is a static timing function mapping each transition to a closed non-empty interval with non-negative rational boundaries; right open infinite boundaries are allowed. For a transition $t \in T$, the boundaries of the interval $D(t)$ are called the earliest firing time (Eft) and latest firing time (Lft) of t

A state of $\mathcal{T N}$ is a pair $S=(M, I)$, where $M$ is a marking and $I: E n(M) \rightarrow \mathbb{R}_{\geq 0}$ is a dynamic timing function. The initial state of $\mathcal{T} \mathcal{N}$ is a pair $S_{0}=\left(M_{0}, I_{0}\right)$, where $M_{0}$ is the initial marking and $I_{0}(t)=0$, for all $t \in \operatorname{En}\left(M_{0}\right)$.

A transition $t$ can fire from a state $S=(M, I)$ if $t \in E n(M)$ and $E f t(t) \leq I(t) \leq L f t(t)$ In the TPN, two types of state changes are possible by:

a) the elapsing of time $\tau \in \mathbb{R}_{\geq 0}$, defined as follows:

$$
(M, I) \stackrel{\tau}{\rightarrow}\left(M, I^{\prime}\right) \text { iff } \forall t^{\prime} \in \operatorname{En}(M): I^{\prime}\left(t^{\prime}\right)=I\left(t^{\prime}\right)+\tau ;
$$

b) the firing of a transition $t \in T$, defined as follows:

$$
(M, I) \stackrel{t}{\rightarrow}\left(M^{\prime}, I^{\prime}\right) \text { iff }\left\{\begin{array}{c}
t \text { can fire from }(M, I), \text { and } \\
M^{\prime}=(M \backslash \bullet t) \cup t \bullet, \text { and } \\
\forall t^{\prime} \in E n\left(M^{\prime}\right): I^{\prime}\left(t^{\prime}\right)=\left\{\begin{array}{cc}
0, & \text { if } \uparrow \text { enabled }\left(t^{\prime}, M, t\right), \\
I\left(t^{\prime}\right), & \text { otherwise, }
\end{array}\right.
\end{array}\right.
$$




\section{where the predicate}

$\uparrow \operatorname{enabled}\left(t^{\prime}, M, t\right)=t^{\prime} \in E n((M \backslash \bullet t) \cup t \bullet) \wedge\left(t^{\prime} \notin E n(M \backslash \bullet t) \vee t=t^{\prime}\right)$

indicates whether we need to reset the clock of $t^{\prime}$ after the firing of $t$ at $M$.

We use the notation $S \stackrel{\sigma}{\rightarrow} S^{\prime}$ iff $\sigma=\overline{t_{1}} \ldots \overline{t_{k}} \in\left(T \cup \mathbb{R}_{\geq 0}\right)^{k}$ and $S=S^{0} \stackrel{\overline{t_{1}}}{\rightarrow} S^{1} \ldots S^{k-1} \stackrel{\overline{t_{k}}}{\rightarrow} S^{k}=$ $S^{\prime}(k \geq 0)$. In this case, $\sigma$ is a run of $\mathcal{T} \mathcal{N}$ from $S\left(\right.$ to $\left.S^{\prime}\right)$, and $S^{\prime}$ is a reachable state of $\mathcal{T} \mathcal{N}$ from $S$. Let $\mathcal{F} \mathcal{S}(\mathcal{T} \mathcal{N})$ be the set of all runs of $\mathcal{T} \mathcal{N}$ from $S_{0}, \mathcal{R} \mathcal{S}(\mathcal{T} \mathcal{N})$ be the set of all reachable states of $\mathcal{T N}$ from $S_{0}$. We write $\operatorname{Untimed}(\sigma)$ to denote the projection $\sigma$ on $T$, i.e. the untimed part of $\sigma$.

Consider some properties of TPNs. We call $\mathcal{T} \mathcal{N}$ safe, iff $M(p) \leq 1$, for all $S=(M, I) \in R S(\mathcal{T N})$ and $p \in P$; contact-free iff whenever $t$ can fire from a state $S=(M, I)$, then $(M \backslash \bullet t) \cap t \bullet=\varnothing$ for all $S \in \mathcal{R} \mathcal{S}(\mathcal{T N} \mathcal{N}) ; T$-restricted iff $\bullet \neq \varnothing$ and $t \bullet \neq \varnothing$ for all transitions in the underlying Petri net.

Notice that the definition of the marking of the underlying PN as a subset, rather than a multiset, of the net places (see Definition 1) ensures that each place has at most one token when the TPN is functioning, i.e. it is safe. This leads to the fact that any transition can be enabled at most once at any marking $M$ and can fire at most once from a corresponding state $(M, I)$. As a consequence, $E n(M)$ is a set, rather than a multiset, of transitions, and the dynamic timing function $I$ is really a function, rather than a relation. The contact-freeness property says that a transition cannot fire from a state, if at least one output place (which is not the input place) of the transition already contains a token at the corresponding marking. In the case when the TPN is not contact-free, after the firing of an enabled transition from a state, two or more tokens can accumulate in the output places of the transition. However, some of the tokens may be lost, as the marking is defined as a subset, rather than a multiset of places. Due to the $T$-restrictedness property, each net transition has at least one input place and at least one output place. This allows us to avoid livelock (useless work) situations as the transitions without input and output places can fire (work) infinitely many times without consuming and producing any tokens (results). So, the above properties facilitate the correct definitions and results concerning TPNs. In what follows, we will consider only safe, contact-free and $T$-restricted TPNs.

Example 1. A (labeled over Act $=\{a, b\}$ ) time Petri net $\widetilde{\mathcal{T N}}$ is shown in Fig. 1. Here, the places are represented by circles and transitions by squares; the names are depicted near the elements. The elements included in the flow relation are connected by arrows, and each place contained in the initial marking is that with a token (bold point). The values of the labeling and static timing functions are printed next to the transitions. It is easy to see that $\widetilde{\mathcal{T N}}$ is really safe, contact-free and $T$-restricted. Show that $\sigma=t_{1} t_{3}(2.3) t_{2}(1.5) t_{3}$ is a run of $\mathcal{T N}$ from $S_{0}$

- $S_{0}=\left(M_{0}, I_{0}\right)$, where $M_{0}=\left\{p_{1}, p_{2}\right\}, E n\left(M_{0}\right)=\left\{t_{1}, t_{3}\right\}$ and $\forall t \in E n\left(M_{0}\right): I_{0}(t)=0$.

- Due to $t_{1} \in \operatorname{En}\left(M_{0}\right)$ and $\operatorname{Eft}\left(t_{1}\right)=0 \leq I_{0}\left(t_{1}\right)=0 \leq \operatorname{Lft}\left(t_{1}\right)=1$, we have that $t_{1}$ can fire from $\left(M_{0}, I_{0}\right)$. Then, $S_{0} \stackrel{t_{1}}{\rightarrow} S_{1}=\left(M_{1}, I_{1}\right)$, where $M_{1}=\left(M_{0} \backslash \bullet t_{1}\right) \cup t_{1} \bullet=\left\{p_{2}, p_{3}\right\}$, $\operatorname{En}\left(M_{1}\right)=\left\{t_{3}\right\}$ and $I_{1}\left(t_{3}\right)=I_{0}\left(t_{3}\right)=0$, because $\uparrow$ enabled $\left(t_{3}, M_{0}, t_{1}\right)=$ false.

- Due to $t_{3} \in \operatorname{En}\left(M_{1}\right)$ and $\operatorname{Eft}\left(t_{3}\right)=0 \leq I_{1}\left(t_{3}\right)=0 \leq \operatorname{Lft}\left(t_{3}\right)=2$, we have that $t_{3}$ can fire from $\left(M_{1}, I_{1}\right)$. Then, $S_{0} \stackrel{t_{1} t_{3}}{\longrightarrow} S_{2}=\left(M_{2}, I_{2}\right)$, where $M_{2}=\left(M_{1} \backslash \bullet t_{3}\right) \cup t_{3} \bullet=\left\{p_{3}, p_{4}\right\}$, $E n\left(M_{2}\right)=\left\{t_{2}\right\}$ and $I_{2}\left(t_{2}\right)=0$, because $\uparrow$ enabled $\left(t_{2}, M_{1}, t_{3}\right)=$ true

- $S_{0} \stackrel{t_{1} t_{3}(2.3)}{\longrightarrow} S_{3}=\left(M_{3}, I_{3}\right)$, where $M_{3}=M_{2}=\left\{p_{3}, p_{4}\right\}, E n\left(M_{3}\right)=\left\{t_{2}\right\}$ and $I_{3}\left(t_{2}\right)=I_{2}\left(t_{3}\right)+$ $2.3=2.3$.

- Due to $t_{2} \in \operatorname{En}\left(M_{3}\right)$ and $\operatorname{Eft}\left(t_{2}\right)=1 \leq I_{3}\left(t_{2}\right)=2.3 \leq \operatorname{Lft}\left(t_{2}\right)=3$, we have that $t_{2}$ can fire from $\left(M_{3}, I_{3}\right)$. Then, $S_{0} \stackrel{t_{1} t_{3}(2.3) t_{2}}{\longrightarrow} S_{4}=\left(M_{4}, I_{4}\right)$, where $M_{4}=\left(M_{3} \backslash \bullet t_{2}\right) \cup t_{2} \bullet=\left\{p_{1}, p_{2}\right\}$,
Virbitskaite I.B., Zubarev A.Yu. Time Causal Processes in Time Petri Nets with Weak Semantics. Trudy ISP RAN/Proc. ISP RAS, vol. 32 ,

$\operatorname{En}\left(M_{4}\right)=\left\{t_{1}, t_{3}\right\}$

$\uparrow$ enabled $\left(t_{3}, M_{3}, t_{2}\right)$

true

- $S_{0} \stackrel{t_{1} t_{3}(2.3) t_{2}(1.5)}{\longrightarrow} S_{5}=\left(M_{5}, I_{5}\right)$, where $M_{5}=M_{4}=\left\{p_{1}, p_{2}\right\}, \operatorname{En}\left(M_{5}\right)=\left\{t_{1}, t_{3}\right\}$ and $I_{5}\left(t_{1}\right)=$ $I_{4}\left(t_{1}\right)+1.5=1.5, I_{5}\left(t_{3}\right)=I_{4}\left(t_{3}\right)+1.5=1.5$

- Due to $t_{3} \in \operatorname{En}\left(M_{5}\right)$ and $E f t\left(t_{3}\right)=0 \leq I_{5}\left(t_{3}\right)=1.5 \leq \operatorname{Lft}\left(t_{3}\right)=2$, we have that $t_{3}$ can fire from $\left(M_{5}, I_{5}\right)$. Then, $S_{0} \stackrel{t_{1} t_{3}(2.3) t_{2}(1.5) t_{3}}{\longrightarrow} S_{6}=\left(M_{6}, I_{6}\right)$, where $M_{6}=\left(M_{5} \backslash \bullet t_{3}\right) \cup t_{3} \cdot=$ $\left\{p_{1}, p_{4}\right\}, \quad E n\left(M_{6}\right)=\left\{t_{1}\right\} \quad$ and $\quad I_{6}\left(t_{1}\right)=I_{5}\left(t_{1}\right)=1.5, \quad$ because $\uparrow$ enabled $\left(t_{1}, M_{5}, t_{3}\right)=$ false.

Therefore, $\sigma=t_{1} t_{3}(2.3) t_{2}(1.5) t_{3}$ is a run of $\mathcal{T} \mathcal{N}$ from $S_{0}$.

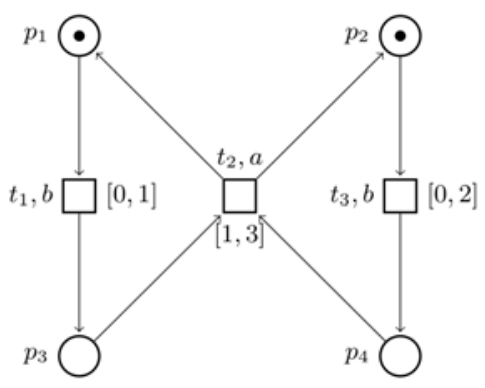

Fig. 1. A time Petri net $\widetilde{\mathcal{T N}}=(\widetilde{\mathcal{N}}, D)$

In order to display that every TPN can be transform into that with natural-valued boundaries of the intervals associated with its transitions, we need a notion of time equivalence. Two TPNs are considered time equivalent if they have the same underlying Petri net, and for each transition, its earliest and latest firing times in the TPNs are either proportional to a non-zero constant or its lates firing times are together equal to infinity.

Definition 3. Two time Petri nets $\mathcal{T} \mathcal{N}_{1}=\left(\mathcal{N}, D_{1}\right)$ and $\mathcal{T} \mathcal{N}_{2}=\left(\mathcal{N}, D_{2}\right)$ are time equivalent iff there exists a non-negative constant $c \neq 0$ such that for any transition $t$ in $\mathcal{N}$ it holds.

- $E f t_{2}(t)=E f t_{1}(t) \cdot c$,

$-L f t_{2}(t)=\left\{\begin{array}{c}\infty, \quad \text { if } L f t_{1}(t)=\infty, \\ L f t_{1}(t) \cdot c_{1}\end{array}\right.$

otherwise.

We next establish that for any TPN there is a time equivalent TPN with time intervals having natural-valued boundaries.

Theorem 1. Given a TPN $\mathcal{T} \mathcal{N}_{1}=\left(\mathcal{N}=\left(P, T, F, M_{0}, L\right), D_{1}\right)$, there exists a TPN $\mathcal{T N}_{2}=$ $\left(\mathcal{N}, D_{2}\right)$, with $D_{2}: T \rightarrow \mathbb{N} \times(\mathbb{N} \cup\{\infty\})$, such that $\mathcal{T} \mathcal{N}_{1}$ and $\mathcal{T} \mathcal{N}_{2}$ are time equivalent. Moreover, for any $\sigma_{1} \in \mathcal{F} \mathcal{S}\left(\mathcal{T} \mathcal{N}_{1}\right)$, there is $\sigma_{2} \in \mathcal{F} \mathcal{S}\left(\mathcal{T} \mathcal{N}_{2}\right)$ with the same transition firings and time elapsings multiplied by a constant $c$, and vice versa.

Proof. Construct the set $\mathcal{D}$ of the denominators of the boundaries from $D_{1}$ as follows: $\mathcal{D}=$ $\left\{n \mid E f t_{1}(t)=\frac{m}{n} ; t \in T ; m, n \in \mathbb{N}_{>0}\right\} \cup\left\{n \mid L f t_{1}(t)=\frac{m}{n} ; t \in T ; m, n \in \mathbb{N}_{>0}\right\} . \quad$ Calculate the least common multiple of the denominators: $c=\left\{\begin{array}{cc}1, & \text { if } \mathcal{D}=\emptyset \\ \operatorname{LCM}(\mathcal{D}), & \text { otherwise }\end{array}\right.$. Due to $c$ being the least common multiple, we have non-negative constant $c \neq 0$. Define $D_{2}: T \rightarrow \mathbb{N} \times(\mathbb{N} \cup\{\infty\})$ as follows: 


\section{- Eft $t_{2}(t)=E f t_{1}(t) \cdot c$, \\ - $L f t_{2}(t)=\left\{\begin{array}{cc}\infty, & \text { if } L f t_{1}(t)=\infty \\ L f t_{1}(t) \cdot c, & \text { otherwise, }\end{array}\right.$ \\ - $D_{2}(t)=\left(E f t_{2}(t), L f t_{2}(t)\right)$}

So, $\mathcal{T} \mathcal{N}_{1}$ and $\mathcal{T} \mathcal{N}_{2}$ are time equivalent.

Take an arbitrary $\sigma_{1}=\overline{t_{1}} \ldots \overline{t_{k}} \in \mathcal{F} \mathcal{S}\left(\mathcal{T} \mathcal{N}_{1}\right)$, with $\left(M_{0}, I_{0}\right) \stackrel{\overline{t_{1}}}{\rightarrow}\left(M_{1}, I_{1}\right) \ldots\left(M_{k-1}, I_{k-1}\right) \stackrel{\overline{t_{k}}}{\rightarrow}\left(M_{k}, I_{k}\right)$. Construct $\sigma_{2}=\overline{t_{1}^{\prime}} \ldots \overline{t_{k}^{\prime}}$ such that $\bar{t}_{l} \in T \Rightarrow \overline{t_{l}^{\prime}}=\bar{t}_{l}$ and $\bar{t}_{l} \in \mathbb{R}_{\geq 0} \Rightarrow \bar{t}_{l}^{\prime}=\bar{t}_{l} \cdot c$, for all $1 \leq i \leq k$. We shall prove that $\sigma_{2} \in \mathcal{F} \mathcal{S}\left(\mathcal{T} \mathcal{N}_{2}\right)$, with $\left(M_{0}^{\prime}, I_{0}^{\prime}\right) \stackrel{\overline{t_{1}^{\prime}}}{\rightarrow}\left(M_{1}^{\prime}, I_{1}^{\prime}\right) \ldots\left(M_{k-1}^{\prime}, I_{k-1}^{\prime}\right) \stackrel{\overline{t_{k}^{\prime}}}{\rightarrow}\left(M_{k}^{\prime}, I_{k}^{\prime}\right)$, by induction on $1 \leq i \leq k$

$i=0$. Then, $M_{0}^{\prime}=M_{0}$, due to $\mathcal{T} \mathcal{N}_{1}$ and $\mathcal{T} \mathcal{N}_{2}$ having the same underlying Petri net; and $I_{0}^{\prime}(t)=0$, for all $t \in \operatorname{En}\left(M_{0}^{\prime}\right)$, due to Definition 2 .

$i>0$. By the induction hypothesis, we have that $\left(M_{0}^{\prime}, I_{0}^{\prime}\right) \stackrel{\overline{t_{1}^{\prime} \ldots \overline{t_{l-1}^{\prime}}}}{\longrightarrow}\left(M_{i-1}^{\prime}, I_{i-1}^{\prime}\right)$. Thanks to the construction of $\sigma_{2}$, we obtain that $M_{j}^{\prime}=M_{j}$, and, hence, $\operatorname{En}\left(M_{j}^{\prime}\right)=\operatorname{En}\left(M_{j}\right)$, for all $0 \leq j \leq i-1$, due to Definition 2. Then, it holds that $\uparrow \operatorname{enabled}\left(t, M_{j-1}^{\prime}, \overline{t_{j}^{\prime}}\right)=\uparrow \operatorname{enabled}\left(t, M_{j-1}, \bar{t}_{j}\right)$, for all $t \in$ $E n\left(M_{j-1}^{\prime}\right)=E n\left(M_{j-1}\right)$ and for all $1 \leq j \leq i$, i.e. the prefixes of $\sigma_{1}$ and $\sigma_{2}$ have the same clock resets for enabled transitions. This implies that $I_{j}^{\prime}(t)=I_{j}(t) \cdot c$, for all $t \in \operatorname{En}\left(M_{j}^{\prime}\right)$ and $0 \leq j \leq i-$ 1 , due to Definition 2 and the construction of $\sigma_{2}$. Show that $\left(M_{i-1}^{\prime}, I_{i-1}^{\prime}\right) \stackrel{\bar{t}_{l}^{\prime}}{\rightarrow}\left(M_{i}^{\prime}, I_{i}^{\prime}\right)$. Two cases are admissible.

1. $\overline{t_{l}^{\prime}} \in \mathbb{R}_{\geq 0}$. Then, $\overline{t_{l}^{\prime}}=\bar{t}_{l} \cdot c$, by the construction of $\sigma_{2}$. As $\sigma_{1} \in \mathcal{F} \mathcal{S}\left(\mathcal{T} \mathcal{N}_{1}\right)$, we have that $M_{i}=$ $M_{i-1}$ and $\forall t \in \operatorname{En}\left(M_{i-1}\right): I_{i}(t)=I_{i-1}(t)+\bar{t}_{l}$, due to Definition 2. Then, it holds that $I_{i}(t) \cdot c=I_{i-1}(t) \cdot c+\overline{t_{l}} \cdot c=I_{i-1}^{\prime}(t)+\overline{t_{l}^{\prime}}=I_{i}^{\prime}(t)$, for all $t \in E n\left(M_{i-1}^{\prime}=M_{i}^{\prime}\right)$, Therefore, $\left(M_{i-1}^{\prime}, I_{i-1}^{\prime}\right) \stackrel{\vec{t}_{l}^{\prime}}{\rightarrow}\left(M_{i}^{\prime}, I_{i}^{\prime}\right)$, according to Definition 2 .

2. $\overline{t_{l}^{\prime}} \in T$. Then, $\overline{t_{l}^{\prime}}=\bar{t}_{t}$, by the construction of $\sigma_{2}$. As $\sigma_{1} \in \mathcal{F} \mathcal{S}\left(\mathcal{T} \mathcal{N}_{1}\right)$, we have that $\bar{t}_{1} \in \operatorname{En}\left(M_{i-1}\right)$ and $E f t_{1}\left(\bar{t}_{l}\right) \leq I_{i-1}\left(\bar{t}_{l}\right) \leq L f t_{1}\left(\bar{t}_{l}\right)$, because $\bar{t}_{1}$ can fire from $\left(M_{i-1}, I_{i-1}\right)$. Hence, we obtain that $\overline{t_{1}^{\prime}} \in E n\left(M_{i-1}^{\prime}\right) \quad$ and $E f t_{2}\left(\overline{t_{l}^{\prime}}\right)=E f t_{1}\left(\bar{t}_{l}\right) \cdot c \leq I_{i-1}\left(\bar{t}_{l}\right) \cdot c=I_{i-1}^{\prime}\left(\bar{t}_{l}^{\prime}\right)=I_{i-1}\left(\bar{t}_{l}\right) \cdot c \leq$ $\left\{\begin{array}{cc}\infty, & \text { if } L f t_{1}\left(\bar{t}_{l}\right)=\infty, \\ L f t_{1}\left(\bar{t}_{l}\right) \cdot c, & \text { otherwise. }\end{array}\right\}=L f t_{2}\left(\bar{t}_{l}^{\prime}\right)$, due to $\mathcal{T} \mathcal{N}_{1}$ and $\mathcal{T} \mathcal{N}_{2}$ being time equivalent with a proportionality constant $c$. So, $\overline{t_{l}^{\prime}}$ can fire from $\left(M_{i-1}^{\prime}, I_{i-1}^{\prime}\right)$. Since $\sigma_{1} \in \mathcal{F} \mathcal{S}\left(\mathcal{T} \mathcal{N}_{1}\right)$, we have that $M_{i}=\left(M_{i-1} \backslash \bullet \bar{t}_{l}\right) \cup \overline{t_{l}} \bullet$ and $\forall t \in \operatorname{En}\left(M_{i}\right): I_{i}(t)=\left\{\begin{array}{cc}0, & \text { if } \uparrow \operatorname{enabled}\left(t, M_{i-1}, \bar{t}_{l}\right) \text {, }\end{array}\right.$ that $M_{i}=\left(M_{i-1} \backslash \bullet \bar{t}_{l}\right) \cup t_{l} \bullet$ and $\forall t \in E n\left(M_{i}\right): I_{i}(t)= \begin{cases}I_{i-1}(t), & \text { otherwise }\end{cases}$ by Definition 2. Then, it holds that $\left(M_{i-1}^{\prime} \backslash \bullet \overline{t_{l}^{\prime}}\right) \cup \overline{t_{l}^{\prime}} \bullet=M_{i}^{\prime}=M_{i}$ and $\forall t \in \operatorname{En}\left(M_{i}^{\prime}\right): I_{i}(t)$. $c=\left\{\begin{array}{cc}0, & \text { if } \uparrow \operatorname{enabled}\left(t, M_{i-1}^{\prime}, \overline{t_{l}^{\prime}}\right), \\ I_{i-1}(t) \cdot c=I_{i-1}^{\prime}(t), & \text { otherwise }\end{array}=I_{i}^{\prime}(t) . \quad\right.$ Therefore, $\left(M_{i-1}^{\prime}, I_{i-1}^{\prime}\right) \stackrel{\overline{t_{l}^{\prime}}}{\rightarrow}\left(M_{i}^{\prime}, I_{i}^{\prime}\right)$, thanks to Definition 2 .

Thus, $\left(M_{0}^{\prime}, I_{0}^{\prime}\right) \stackrel{\sigma_{2}}{\rightarrow}\left(M_{k}^{\prime}, I_{k}^{\prime}\right)$, i.e. $\sigma_{2} \in \mathcal{F} \mathcal{S}\left(\mathcal{T} \mathcal{N}_{2}\right)$.

Reasoning analogously, we can show that for each $\sigma_{2} \in \mathcal{F} \mathcal{S}\left(\mathcal{T} \mathcal{N}_{2}\right)$, there exists $\sigma_{1} \in \mathcal{F} \mathcal{S}\left(\mathcal{T} \mathcal{N}_{1}\right)$ such that $\sigma_{1}$ and $\sigma_{2}$ have the same untimed part.

Example 2. Consider the PN $\widetilde{\mathcal{N}}$ whose structure is shown in Fig. 1. Define the TPN $\overline{\mathcal{T} \mathcal{N}_{1}}=\left(\widetilde{\mathcal{N}}, D_{1}\right)$, with $D_{1}\left(t_{1}\right)=\left[0, \frac{1}{4}\right], D_{1}\left(t_{2}\right)=\left[\frac{1}{4}, \frac{3}{4}\right], D_{1}\left(t_{3}\right)=\left[0, \frac{1}{2}\right]$. Construct a TPN $\widetilde{\mathcal{T N} \mathcal{N}_{2}}=\left(\widetilde{\mathcal{N}}, D_{2}\right)$, with $D_{2}: T \rightarrow \mathbb{N} \times(\mathbb{N} \cup\{\infty\})$ such that $\frac{\overline{\mathcal{T}} \mathcal{N}_{1}}{1}$ and $\widetilde{\mathcal{T} \mathcal{N}_{2}}$ are time equivalent. Define the set $\mathcal{D}$ as follow: $\mathcal{D}=\left\{n \mid E f t_{1}(t)=\frac{m}{n}, t \in T, m, n \in \mathbb{N}_{>0}\right\} \cup\left\{n \mid L f t_{1}(t)=\frac{m}{n}, t \in T, m, n \in \mathbb{N}_{>0}\right\}=\{2,4\}$.
Let $c=\operatorname{LCM}(\mathcal{D})=\operatorname{LCM}(2,4)=4, \quad D_{2}\left(t_{1}\right)=\left[E f t_{1}\left(t_{1}\right) * c=0 * 4, L f t_{1}\left(t_{1}\right) * c=\frac{1}{4} * 4\right]=$ $[0,1], D_{2}\left(t_{2}\right)=\left[E f t_{1}\left(t_{2}\right) * c=\frac{1}{4} * 4, L f t_{1}\left(t_{2}\right) * c=\frac{3}{4} * 4\right]=[1,3]$, and $D_{2}\left(t_{3}\right)=\left[E f t_{1}\left(t_{3}\right) *\right.$ $\left.c=0 * 4, L f t_{1}\left(t_{3}\right) * c=\frac{1}{2} * 4\right]=[0,2]$. Then, the time intervals of $\overline{\mathcal{T N}_{2}}$ have natural-valued boundaries. Moreover, $\overline{\widetilde{\mathcal{T N}}_{1}}$ and $\overline{\mathcal{T} \mathcal{N}_{2}}$ are time equivalent, because the static functions in the TPNs are proportional to the non-zero constant 4 . As a consequence, for example, for the run $\sigma_{1}=$ $t_{1} t_{3}(0.25) t_{2}(0.125) t_{3}$ of $\overline{\mathcal{T} \mathcal{N}_{1}}$, there exists the run $\sigma_{2}=t_{1} t_{3}(1) t_{2}(0.5) t_{3}$ of $\overline{\mathcal{T} \mathcal{N}_{2}}$, with time elapsings that are four longer times. Conversely, for the run $\sigma_{2}$ of $\overline{\mathcal{T} \mathcal{N}_{2}}$, there is the run $\sigma_{1}$ of $\overline{\mathcal{T} \mathcal{N}_{1}}$ with time elapsings that are four times shorter.

So, if two time Petri nets are time equivalent, then each run of the one TPN can be obtained from some run of the other TPN with the same untimed part and with time elapsings multiplied by the proportionality constant. In other words, time in the one TPN goes slower (or faster) than in the other TPN. With that, it is worth stressing that the TPNs have the same behavioral properties (e.g., safeness, liveness, marking reachability, etc.).

In the sequel, we will consider only TPNs with time intervals having natural-valued boundaries.

\section{State Space Discretization for $\mathcal{T} \mathcal{N}$}

In this section, we demonstrate that in the TPN for any run there exists a run with the same untimed part and with natural-value time (and even unit time) elapsings.

For the TPN $\mathcal{T N}$, let $\widehat{\mathcal{F S}}(\mathcal{T N})$ be the set of all runs from $\mathcal{F} \mathcal{S}(\mathcal{T N})$ of the form: $\hat{\sigma}=$ $\tau_{0} t_{1} \tau_{1} \ldots \tau_{k-1} t_{k} \tau_{k}$, i.e. time elapsings and transition firings alternate in the runs. So, in $\mathcal{T N}$ for the run $\hat{\sigma} \in \widehat{\mathcal{F} S}(\mathcal{T N})$, we have the following changes in states: $\left(M_{0}, I_{0}\right) \stackrel{\tau_{0}}{\rightarrow}\left(M_{0}, I_{0}^{\prime}\right) \stackrel{t_{1}}{\rightarrow}\left(M_{1}, I_{1}\right) \stackrel{\tau_{1}}{\rightarrow}\left(M_{1}, I_{1}^{\prime}\right) \ldots\left(M_{k-1}, I_{k-1}\right) \stackrel{\tau_{k-1}}{\rightarrow}\left(M_{k-1}, I^{\prime}{ }_{k-1}\right) \stackrel{t_{k}}{\rightarrow}\left(M_{k}, I_{k}\right) \stackrel{\tau_{k}}{\rightarrow}\left(M_{k}, I_{k}^{\prime}\right)$ As we will see later (in the proof of Corollary 2$)$, any run from $\mathcal{F} \mathcal{S}(\mathcal{T N} \mathcal{N})$ can be represented in the above form. Define the set $\widehat{\mathcal{U F \mathcal { S }}}(\mathcal{T} \mathcal{N})=\{\operatorname{Untimed}(\hat{\sigma}) \mid \hat{\sigma} \in \widehat{\mathcal{F S}}(\mathcal{T} \mathcal{N})\}$

Next, for an untimed sequence from $\widehat{\mathcal{U F} \mathcal{S}}(\mathcal{T} \mathcal{N})$, we construct the parametric run (which is, in fact, a modification of a run, with variables instead of the time elapsing values) and a set of conditions on the values of these variables, by induction of the number of the variables. At each induction step, we define a prefix of the parametric run and the conditions for the values of its variables, increasing the run's length by one variable.

Definition 4. Let $\mathcal{T} \mathcal{N}=\left(\left(P, T, F, M_{0}, L\right), D\right)$ be a time Petri net, $t_{1} \ldots t_{k} \in \widehat{U \mathcal{F} \mathcal{S}}(\mathcal{T N})$ and $X=\left\{x_{0}, \ldots, x_{k}\right\}$ be a set of variables. We construct a finite sequence of the tuples of the form $\left(\omega_{i}, B_{\omega_{i}}, M_{\omega_{i}}, I^{\prime} \omega_{i}\right)$, by induction on $0 \leq i \leq k$.

$i=0$. Then,

$-\omega_{0}=x_{0}$

$-B_{\omega_{0}}=\emptyset$

- $M_{\omega_{0}}=M_{0}$

- $I^{\prime}{ }_{\omega_{0}}(t)=x_{0}$, for all $t \in E n\left(M_{\omega_{0}}\right)$.

$i>0$. Assume that $\left(\omega_{i-1}, B_{\omega_{i-1}}, M_{\omega_{i-1}}, I^{\prime} \omega_{i-1}\right)$ is already constructed. Then,

- $\omega_{i}=\omega_{i-1} t_{i} x_{i}$

- $B_{\omega_{i}}=B_{\omega_{i-1}} \cup\left\{E f t\left(t_{i}\right) \leq I^{\prime} \omega_{i-1}\left(t_{i}\right) \leq L f t\left(t_{i}\right)\right\}$

- $M_{\omega_{i}}=\left(M_{\omega_{i-1}} \backslash \bullet t_{i}\right) \cup t_{i} \bullet$ 
- $I^{\prime} \omega_{i}(t)=\left\{\begin{array}{cc}x_{i}, & \text { if } \uparrow \text { enabled }\left(t, M_{\omega_{i-1}}, t_{i}\right), \\ I^{\prime}{ }_{\omega_{i-1}}(t)+x_{i}, & \text { otherwise, }\end{array}\right.$ for all $t \in \operatorname{En}\left(M_{\omega_{i}}\right)$.

Then, $\omega=\omega_{k}=x_{0} t_{1} x_{1} \ldots t_{k} x_{k}$ is a parametric run of $\mathcal{T} \mathcal{N}$, and $B_{\omega}=B_{\omega_{k}} \cup\left\{0 \leq x_{k}<\infty\right\}$ is the set of conditions on the values of the variables from $X$.

We use $\mathcal{B}_{\omega}=\left\{I^{\prime} \omega_{i-1}\left(t_{i}\right) \mid\left(E f t\left(t_{i}\right) \leq I^{\prime}{ }_{\omega_{i-1}}\left(t_{i}\right) \leq \operatorname{Lft}\left(t_{i}\right)\right) \in B_{\omega}, 1 \leq i \leq k\right\} \cup\left\{x_{k}\right\}$ to denote the set of the variable parts of the inequalities from $B_{\omega}$.

Example 3. Contemplate the TPN $\widetilde{\mathcal{T N}}=\left(\left(P, T, F, M_{0}, L\right), D\right)$, shown in Fig. 1. For the transition sequence Untimed $(\hat{\sigma})=t_{1} t_{3} t_{2}$, obtained from the run $\hat{\sigma}=(0.5) t_{1}(0.5) t_{3}(2.3) t_{2}(1.7)$ of $\widetilde{\mathcal{T N}}$, we construct the sequence of the following tuples $\left(\omega_{i}, B_{\omega_{i}}, M_{\omega_{i}}, I^{\prime} \omega_{i}\right)$, with $0 \leq i \leq 3$.

- $i=0$. Set $\omega_{0}=x_{0} ; B_{\omega_{0}}=\emptyset ; M_{\omega_{0}}=M_{0}=\left\{p_{1}, p_{2}\right\} ; \quad E n\left(M_{\omega_{0}}\right)=\left\{t_{1}, t_{3}\right\} ;$ and $I^{\prime}{ }_{\omega_{0}}\left(t_{1}\right)=$ $I^{\prime} \omega_{0}\left(t_{3}\right)=x_{0}$.

$-i=1$. Set $\omega_{1}=\omega_{0} t_{1} x_{1}=x_{0} t_{1} x_{1}$

$B_{\omega_{1}}=B_{\omega_{0}} \cup\left\{\operatorname{Eft}\left(t_{1}\right)=0 \leq I^{\prime} \omega_{0}\left(t_{1}\right) \leq \operatorname{Lft}\left(t_{1}\right)=1\right\}=B_{\omega_{0}} \cup\left\{0 \leq x_{0} \leq 1\right\} ;$

$M_{\omega_{1}}=\left(M_{\omega_{0}} \backslash \cdot t_{1}\right) \cup t_{1} \bullet=\left\{p_{3}, p_{2}\right\}$ and $\operatorname{En}\left(M_{\omega_{1}}\right)=\left\{t_{3}\right\}$;

$I^{\prime}{ }_{\omega_{1}}\left(t_{3}\right)=I^{\prime}{ }_{\omega_{0}}\left(t_{3}\right)+x_{1}=x_{0}+x_{1}$, as $\uparrow \operatorname{enabled}\left(t_{3}, M_{\omega_{1}}, t_{1}\right)$ is false

- $i=2$. Set $\omega_{2}=\omega_{1} t_{3} x_{2}=x_{0} t_{1} x_{1} t_{3} x_{2}$;

$B_{\omega_{2}}=B_{\omega_{1}} \cup\left\{\operatorname{Eft}\left(t_{3}\right)=0 \leq I^{\prime} \omega_{1}\left(t_{3}\right) \leq L f t\left(t_{3}\right)=2\right\}=B_{\omega_{1}} \cup\left\{0 \leq x_{0}+x_{1} \leq 2\right\} ;$

$M_{\omega_{2}}=\left(M_{\omega_{1}} \backslash \cdot t_{3}\right) \cup t_{3} \cdot=\left\{p_{3}, p_{4}\right\}$ and $E n\left(M_{\omega_{2}}\right)=\left\{t_{2}\right\}$

$I^{\prime} \omega_{2}\left(t_{2}\right)=x_{2}$, as $\uparrow$ enabled $\left(t_{2}, M_{\omega_{2}}, t_{3}\right)$ is true.

- $i=3$. Set $\omega_{3}=\omega_{2} t_{2} x_{3}=x_{0} t_{1} x_{1} t_{3} x_{2} t_{2} x_{3}$;

$B_{\omega_{3}}=B_{\omega_{2}} \cup\left\{E f t\left(t_{2}\right)=1 \leq I^{\prime} \omega_{2}\left(t_{2}\right) \leq L f t\left(t_{2}\right)=3\right\}=B_{\omega_{2}} \cup\left\{1 \leq x_{2} \leq 3\right\}$

$M_{\omega_{3}}=\left(M_{\omega_{2}} \backslash \bullet t_{2}\right) \cup t_{2} \bullet=\left\{p_{1}, p_{2}\right\}$ and $\operatorname{En}\left(M_{\omega_{3}}\right)=\left\{t_{1}, t_{3}\right\}$;

$I^{\prime} \omega_{3}\left(t_{1}\right)=I^{\prime} \omega_{3}\left(t_{3}\right)=x_{3}$, as $\uparrow \operatorname{enabled}\left(t_{1}, M_{\omega_{3}}, t_{2}\right)$ and $\uparrow \operatorname{enabled}\left(t_{3}, M_{\omega_{3}}, t_{2}\right)$ are true.

Then, the parametric run has the form $\omega=\omega_{3}=x_{0} t_{1} x_{1} t_{3} x_{2} t_{2} x_{3}$, where $X=\left\{x_{0}, \ldots, x_{k}\right\}$ is the set of the real variables. Moreover, it holds that

$$
B_{\omega}=B_{\omega_{3}}=\left\{\begin{array}{c}
0 \leq x_{0} \leq 1, \\
0 \leq x_{0}+x_{1} \leq 2, \\
1 \leq x_{2} \leq 3, \\
0 \leq x_{3}<\infty
\end{array}\right\} \text {, and }
$$

$\mathcal{B}_{\omega}=\left\{x_{0}, x_{0}+x_{1}, x_{2}, x_{3}\right\}$.

A function $\beta: X=\left\{x_{0}, \ldots, x_{k}\right\} \rightarrow \mathbb{R}_{\geq 0}$ is called assignment of $\omega$. We write $[\omega]_{\beta}\left(\left[I^{\prime} \omega_{i-1}\left(t_{i}\right)\right]_{\beta}\right)$ for a parametric run $\omega$ (for the value of a linear function $I^{\prime}{ }_{\omega_{i-1}}\left(t_{i}\right) \in \mathcal{B}_{\omega}$ ) under the assignment $\beta$. The mapping $\beta$ is a solution of $B_{\omega}$ iff $E f t\left(t_{i}\right) \leq\left[I^{\prime} \omega_{i-1}\left(t_{i}\right)\right]_{\beta} \leq \operatorname{Lft}\left(t_{i}\right)$, for all $I_{\omega_{i-1}}^{\prime}\left(t_{i}\right) \in \mathcal{B}_{\omega}$.

Example 4. Consider the parametric run $\omega=x_{0} t_{1} x_{1} t_{3} x_{2} t_{2} x_{3}$, the set $\mathcal{B}_{\omega}=\left\{x_{0}, x_{0}+x_{1}, x_{2}, x_{3}\right\}$, from Example 3, and an assignment $\beta:\left\{x_{0}, \ldots, x_{3}\right\} \rightarrow \mathbb{R}_{\geq 0}$ such that $\beta\left(x_{0}\right)=0.7, \beta\left(x_{1}\right)=$ $0.3, \beta\left(x_{2}\right)=1.4, \beta\left(x_{3}\right)=2$. Then, we obtain that $[\omega]_{\beta}=(0.7) t_{1}(0.3) t_{3}(1.4) t_{2}(2)$. Moreover, we get: $\operatorname{Eft}\left(t_{1}\right)=0 \leq\left[x_{0}\right]_{\beta}=0.7 \leq L f t\left(t_{1}\right)=1, \operatorname{Eft}\left(t_{3}\right)=0 \leq\left[x_{0}+x_{1}\right]_{\beta}=1 \leq L f t\left(t_{3}\right)=$ $2, \operatorname{Eft}\left(t_{2}\right)=1 \leq\left[x_{2}\right]_{\beta}=1.4 \leq \operatorname{Lft}\left(t_{2}\right)=3$. Therefore, $\beta$ is a solution of $B_{\omega}$

Lemma 1. Let $\mathcal{T N}$ be a TPN, $\omega=x_{0} t_{1} x_{1} \ldots t_{k} x_{k}$ be a parametric run of $\mathcal{T N}$, and $B_{\omega}$ be the set of conditions on the values of the variables $x_{0}, x_{1}, \ldots, x_{k}$. If $\beta$ is a solution of $B_{\omega}$, then $[\omega]_{\beta} \in$ $\widehat{\mathcal{F S}}(\mathcal{T N})$. Moreover, for any $\left.I^{\prime}{ }_{\omega_{i-1}}\left(t_{i}\right) \in \mathcal{B}_{\omega},{ }^{\prime} I^{\prime}{ }{ }_{i-1}\left(t_{i}\right)\right]_{\beta}$ is the value of $I^{\prime}{ }_{i-1}\left(t_{i}\right)$ in the run $[\omega]_{\beta}$, when $\mathcal{T N}$ functions along the run.

Proof. See Appendix.

Next, for an arbitrary run $\hat{\sigma}$ from $\widehat{\mathcal{F S}}(\mathcal{T} \mathcal{N})$ with real-value time elapsings, we construct a naturalvalue assignment $\beta_{\omega}$ to the variables in the corresponding parametric run $\omega$ of $\mathcal{T} \mathcal{N}$, by induction on the number of the variables in $\omega$ (i.e. the number of time elapsings in the run $\hat{\sigma}$ ). Starting from the end of the run $\hat{\sigma}$, at each induction step, we round, the value of the corresponding time elapsing of $\hat{\sigma}$, down or up to a natural number nearest to the value and agreed with the values of the other time elapsings in $\hat{\sigma}$.

Definition 5. Let $\mathcal{T} \mathcal{N}$ be a TPN, $\tau_{0} t_{1} \tau_{1} \ldots t_{k} \tau_{k} \in \widehat{\mathcal{F} \mathcal{S}}(\mathcal{T N} \mathcal{N}), \omega=x_{0} t_{1} x_{1} \ldots t_{k} x_{k}$ be the parametric run of $\mathcal{T} \mathcal{N}, B_{\omega}$ be the set of conditions on the values of the variables from $X=\left\{x_{0}, \ldots, x_{k}\right\}$, and $\mathcal{B}_{\omega}$ be the set of the variable parts of the inequalities from $B_{\omega}$. We construct a sequence of functions $\beta_{i}: X \rightarrow \mathbb{R}_{\geq 0}$ by induction on $0 \leq i \leq k$.

$i=0$. Then, for all $0 \leq j \leq k$,

$$
\beta_{0}\left(x_{j}\right)=\left\{\begin{array}{cc}
\left\lfloor{ }^{\tau_{j}}\right\rfloor & \text { if } j=k, \\
\tau_{j} & \text { otherwise }
\end{array} .\right.
$$

$i>0$. In the construction of $\beta_{i}$, we use auxiliary functions defined for all $0 \leq j \leq k$ as follows:

$$
\begin{gathered}
\underline{\beta_{i}}\left(x_{j}\right)=\left\{\begin{array}{c}
\left\lfloor\beta_{i-1}\left(x_{j}\right)\right] \quad \text { if } j=k-i, \\
\beta_{i-1}\left(x_{j}\right) \quad \text { otherwise; }
\end{array} \text { and } \overline{\beta_{i}}\left(x_{j}\right)=\left\{\begin{array}{cc}
{\left[\beta_{i-1}\left(x_{j}\right)\right] \quad \text { if } j=k-i,} \\
\beta_{i-1}\left(x_{j}\right) \quad \text { otherwise. }
\end{array}\right.\right. \\
\text { If } \exists I^{\prime} \omega_{l-1}\left(t_{l}\right) \in \mathcal{B}_{\omega}(1 \leq l \leq k) \text { such that }\left[\left[I^{\prime} \omega_{l-1}\left(t_{l}\right)\right]_{\beta_{i}}\right]<\left\lfloor\left[I^{\prime} \omega_{l-1}\left(t_{l}\right)\right]_{\beta_{0}}\right], \\
\text { then } \beta_{i}=\overline{\beta_{i}}, \text { else } \beta_{i}=\underline{\beta_{i} .}
\end{gathered}
$$

Define a natural-value assignment $\beta_{\omega}: X \rightarrow \mathbb{N}$ as follows: $\beta_{\omega}=\beta_{k}$.

Example 5. Consider the TPN $\widetilde{\mathcal{T N}}$ from Example 1, the run $\hat{\sigma}=(0.5) t_{1}(0.5) t_{3}(2.3) t_{2}(1.7) \in$ $\widehat{\mathcal{F} S}(\overrightarrow{\mathcal{T N}})$, the parametric run $\omega=x_{0} t_{1} x_{1} t_{3} x_{2} t_{2} x_{3}$ and the set $\mathcal{B}_{\omega}=\left\{x_{0}, x_{0}+x_{1}, x_{2}, x_{3}\right\}$ from Example 3. Using Definition 5, construct the sequence of the assignments $\beta_{i}$ by induction on $0 \leq$ $i \leq 3$.

- $i=0$. We set $\beta_{0}\left(x_{j}\right)$, for all $0 \leq j \leq k=3$, as follows: $\beta_{0}\left(x_{0}\right)=\tau_{0}=0.5, \beta_{0}\left(x_{1}\right)=\tau_{1}=$ $0.5, \beta_{0}\left(x_{2}\right)=\tau_{2}=2.3$, and $\beta_{0}\left(x_{3}\right)=\left\lfloor\tau_{3}=1.7\right\rfloor=1$, because $j=k=3$.

- $i=1$. Construct auxiliary functions $\underline{\beta}_{1}\left(x_{j}\right)$ and $\overline{\beta_{1}}\left(x_{j}\right)$, for all $0 \leq j \leq k=3$, as follows: $\beta_{1}\left(x_{0}\right)=\overline{\beta_{1}}\left(x_{0}\right)=\beta_{0}\left(x_{0}\right)=0.5, \quad \beta_{1}\left(x_{1}\right)=\overline{\beta_{1}}\left(x_{1}\right)=\beta_{0}\left(x_{1}\right)=0.5, \beta_{1}\left(x_{3}\right)=\overline{\beta_{1}}\left(x_{3}\right)=$ $\beta_{0}\left(x_{3}\right)=1$, and $\underline{\beta_{1}}\left(x_{2}\right)=\left\lfloor\beta_{0}\left(x_{2}\right)\right\rfloor=2, \overline{\beta_{1}}\left(x_{2}\right)=\left\lceil\beta_{0}\left(x_{2}\right)\right\rceil=3$, because $j=k-i=2$.

Moreover, we get: $\left[\left[x_{0}\right]_{\beta_{1}}\right]=1 \geq\left\lfloor\left[x_{0}\right]_{\beta_{0}}\right\rfloor=0, \quad\left[\left[x_{0}+x_{1}\right]_{\beta_{1}}\right\rceil=1 \geq\left\lfloor\left[x_{0}+x_{1}\right]_{\beta_{0}}\right\rfloor=1$, $\left\lceil\left[x_{2}\right]_{\beta_{1}}\right\rceil=2 \geq\left\lfloor\left[x_{2}\right]_{\beta_{0}}\right\rfloor=2$. Then, $\beta_{1}=\underline{\beta_{1}}$.

- $i=2$. Construct $\underline{\beta_{2}}\left(x_{j}\right)$ and $\overline{\beta_{2}}\left(x_{j}\right)$, with $0 \leq j \leq 3$, as follows: $\underline{\beta_{2}}\left(x_{0}\right)=\overline{\beta_{2}}\left(x_{0}\right)=\beta_{1}\left(x_{0}\right)=$ 


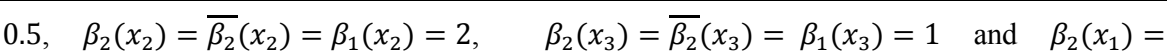
$\left\lfloor\beta_{1}\left(x_{1}\right)\right\rfloor=0, \overline{\beta_{2}}\left(x_{1}\right)=\left\lceil\beta_{1}\left(x_{1}\right)\right\rceil=1$, because $j=k-i=1$.

Moreover, we get: $\left\lceil\left[x_{0}\right]_{\underline{\beta_{2}}}\right\rceil=1 \geq\left\lfloor\left[x_{0}\right]_{\beta_{0}}\right\rfloor=0,\left\lceil\left[x_{0}+x_{1}\right]_{\beta_{2}}\right\rceil=1 \geq\left\lfloor\left[x_{0}+x_{1}\right]_{\beta_{0}}\right\rfloor=1$, $\left[\left[x_{2}\right]_{\beta_{2}}\right]=2 \geq\left\lfloor\left[x_{2}\right]_{\beta_{0}}\right\rfloor=2$. Then, $\beta_{2}=\beta_{2}$.

- $i=3$. Construct $\beta_{3}\left(x_{j}\right)$ and $\overline{\beta_{3}}\left(x_{j}\right)$, with $0 \leq j \leq 3$, as follows: $\beta_{3}\left(x_{1}\right)=\overline{\beta_{3}}\left(x_{1}\right)=\beta_{2}\left(x_{1}\right)=$ $0, \beta_{3}\left(x_{2}\right)=\overline{\beta_{3}}\left(x_{2}\right)=\beta_{2}\left(x_{2}\right)=2, \beta_{3}\left(x_{3}\right)=\overline{\beta_{3}}\left(x_{3}\right)=\beta_{2}\left(x_{3}\right)=1$ and $\beta_{3}\left(x_{0}\right)=\left\lfloor\beta_{2}\left(x_{0}\right)\right\rfloor=$ $0, \overline{\beta_{3}}\left(x_{0}\right)=\left\lceil\beta_{2}\left(x_{0}\right)\right\rceil=1$, because $j=k-i=0$.

Moreover, we have $\left\lceil\left[x_{0}\right]_{\beta_{3}}\right\rceil=0 \geq\left\lfloor\left[x_{0}\right]_{\beta_{0}}\right\rfloor=0,\left\lceil\left[x_{0}+x_{1}\right]_{\beta_{3}}\right\rceil=0<\left\lfloor\left[x_{0}+x_{1}\right]_{\beta_{0}}\right\rfloor=1$, $\left\lceil\left[x_{2}\right]_{\underline{\beta_{3}}}\right]=2 \geq\left\lfloor\left[x_{2}\right]_{\beta_{0}}\right\rfloor=\overline{2}$. Then, there exists $I^{\prime} \omega_{1}\left(t_{3}\right) \in \underline{\mathcal{B}_{\omega}}$ such that $\left[\left[I^{\prime} \omega_{1}\left(t_{3}\right)\right]_{\underline{\beta_{3}}}\right\rceil<$ I[I $\left.\left[I^{\prime} \omega_{1}\left(t_{3}\right)\right]_{\beta_{0}}\right\rfloor$. Therefore, $\beta_{3}=\overline{\beta_{3}}$.

Then, $\beta_{\omega}=\beta_{3}$, and we obtain the sequence $[\omega]_{\beta_{\omega}}=(1) t_{1}(0) t_{3}(2) t_{2}(1)$.

Next, we show that the assignment $\beta_{\omega}$ is a solution of $\omega$, i.e. $\beta_{\omega}$ satisfies the inequalities from $B_{\omega}$. Proposition 1. $\beta_{\omega}$ is a solution of $B_{\omega}$.

Proof. See Appendix.

Thanks to Lemma 1 and Proposition 1, the theorem below follows immediately.

Theorem 2. Let $\mathcal{T N}$ be a time Petri net and $\omega=x_{0} t_{1} x_{1} \ldots t_{k} x_{k}$ be a parametric run of $\mathcal{T N}$. Then, there exists a mapping $\beta_{\omega}: X=\left\{x_{0}, \ldots, x_{k}\right\} \rightarrow \mathbb{N}$ such that $[\omega]_{\beta_{\omega}} \in \widehat{\mathcal{F} \mathcal{S}}(\mathcal{T} \mathcal{N})$.

Proof. Consider the mapping $\beta_{\omega}: X=\left\{x_{0}, \ldots, x_{k}\right\} \rightarrow \mathbb{N}$ from Definition 5. By Proposition 1, $\beta_{\omega}$ is a solution of $B_{\omega}$, and, moreover, $[\omega]_{\beta_{\omega}} \in \widehat{\mathcal{F} \mathcal{S}}(\mathcal{T} \mathcal{N})$, due to Lemma 1 .

We are now ready to show that in the TPN for any run, there exists a run with the same untimed part and with unit time elapsings.

Corollary 2. Let $\mathcal{T N}$ be a time Petri net and $\sigma \in \mathcal{F} \mathcal{S}(\mathcal{T N})$. Then, there is $\sigma^{\prime} \in \mathcal{F} \mathcal{S}(\mathcal{T N})$ with unit-value time elapsings such that $\operatorname{Untimed}(\sigma)=\operatorname{Untimed}\left(\sigma^{\prime}\right)$.

Proof. Due to Definition 2, we obtain the following properties for time elapsings:

a) $S \stackrel{0}{\rightarrow} S$

b) if $S \stackrel{\tau}{\rightarrow} S^{\prime}$ and $S^{\prime} \stackrel{\tau^{\prime}}{\rightarrow} S^{\prime \prime}$, with $\tau, \tau^{\prime} \in \mathbb{R}_{\geq 0}$, then $S \stackrel{\tau+\tau^{\prime}}{\rightarrow} S^{\prime \prime}$;

c) if $S \stackrel{\tau}{\rightarrow} S^{\prime}$, then for every $\tau^{\prime}, \tau^{\prime \prime} \in \mathbb{R}_{\geq 0}$ such that $\tau=\tau^{\prime}+\tau^{\prime \prime}, S \stackrel{\tau^{\prime}}{\rightarrow} S^{\prime \prime} \stackrel{\tau^{\prime \prime}}{\rightarrow} S^{\prime}$ for some $S^{\prime \prime}$.

By items a) and b), there exists $\hat{\sigma} \in \widehat{\mathcal{F} S}(\mathcal{T} \mathcal{N})$ such that $\operatorname{Untimed}(\sigma)=\operatorname{Untimed}(\hat{\sigma})$, thanks to $\sigma$ being a finite sequence. Due to Theorem 2 , there is a run $\widehat{\sigma^{\prime}} \in \widehat{\mathcal{F S}}(\mathcal{T} \mathcal{N})$ with natural-value time elapsings such that $\operatorname{Untimed}(\hat{\sigma})=\operatorname{Untimed}\left(\widehat{\sigma}^{\prime}\right)$. Thanks to c), we can construct $\sigma^{\prime} \in \mathcal{F S}(\mathcal{T} \mathcal{N})$ with unit time elapsings such that Untimed $\left(\widehat{\sigma}^{\prime}\right)=\operatorname{Untimed}\left(\sigma^{\prime}\right)$. Therefore, we obtain that $\operatorname{Untimed}(\sigma)=\operatorname{Untimed}\left(\sigma^{\prime}\right)$.

$\square$

Thanks to Corollary 2, in the sequel, we will consider time Petri nets with unit time elapsings (denoted by $\sqrt{ }$ ).

\section{Time Processes of $\mathcal{T N}$}

In this section, the concept of causality-based net process is presented and studied in the context of TPNs with weak semantics. We start with definitions related to casual nets that contain events and conditions connected by causal dependence and concurrency (absence of causality).

Definition 6. A (labeled over Act $\cup\{$ tick $\})$ casual net is a finite, acyclic net $T N=(B, E, G, l)$

with a set $\mathrm{B}$ of conditions; a set $\mathrm{E}$ of events; a flow relation $G \subseteq(B \times E) \cup(E \times B)$ such that $|b \bullet| \leq 1 \wedge|\bullet b| \leq 1$, for all $b \in B$; a labeling function $l: E \rightarrow$ Act $\cup\{$ tick $\}$.

Informally speaking, the «tick» label means a clock ticking.

Casual nets $T N=(B, E, G, l)$ and $T N^{\prime}=\left(B^{\prime}, E^{\prime}, G^{\prime}, l^{\prime}\right)$ are called isomorphic (denoted $T N \simeq$ $T N^{\prime}$ ) iff there exists a bijective mapping $\gamma: B \cup E \rightarrow B^{\prime} \cup E^{\prime}$ such that:

- $\gamma(B)=B^{\prime}$ and $\gamma(E)=E^{\prime}$;

- $x G y \Leftrightarrow \gamma(x) G^{\prime} \gamma(y)$, for all $x, y \in B \cup E$;

- $l(e)=l^{\prime}(\gamma(e))$, for all $e \in E$.

Introduce auxiliary notions and notations for the casual net $T N=(B, E, G, l)$.

The set $\bullet b(b \bullet)$ is associated with a single event, for any $b \in B$. Let $\bullet T=\{b \in B \mid \bullet b=\varnothing\}$.

Define $\prec=G^{+}, \preccurlyeq=G^{*}$ (causality). A subset $E^{\prime} \subseteq E$ is a downward closed set of events iff $e \in E^{\prime}$ implies $e^{\prime} \in E^{\prime}$, for all $e^{\prime} \prec e$. In this case, $\operatorname{Cut}\left(E^{\prime}\right)=\left(\cdot T N \cup E^{\prime} \bullet\right) \backslash \cdot E^{\prime}$.

A subset $B^{\prime} \subseteq B$ is a co-set (a subset of concurrent conditions) iff $\neg\left(b \prec b^{\prime}\right)$ and $\neg\left(b^{\prime} \prec b\right)$, for all $b, b^{\prime} \in B^{\prime}$. A cut is a maximal (w.r.t. set inclusion) co-set.

A sequence $\rho=e_{1} \ldots e_{n}(n \geq 0)$ of events is a linearization of $T N$ [5] if each event of $T N$ appears in the sequence exactly once, and the following holds: $e_{i} \prec e_{j} \Rightarrow i<j$, for all $1 \leq i, j \leq n$. For a linearization $\rho=e_{1} \ldots e_{n}(n \geq 0)$ of $T N$, define the following:

- $\rho_{0}$ is the empty sequence and $\rho_{k}=e_{1} \ldots e_{k}(1 \leq k \leq n)$;

- $\quad E_{0}=\varnothing$ and $E_{k}=\cup_{1 \leq i \leq k} e_{i}(1 \leq k \leq n)$.

By the construction of the linearization, $E_{k}$ is a downward closed set of events, for all $0 \leq k \leq n$. As we will see later (in Lemma 2), $\operatorname{Cut}\left(E_{k}\right)$ is a cut, for all $1 \leq k \leq n$.

Informally speaking, a linearization is an interleaving representation of a "computation" of $T N$ and the value of the function Cut of any prefix of the linearization is a "marking" of $T N$, reachable after occurring the events from the prefix.

Example 6. Fig. 2 shows a causal net $\widetilde{T N}=(B, E, G, l)$, with $B=\left\{b_{1}, \ldots, b_{10}\right\} ; \quad E=$ $\left\{e_{1}, \ldots, e_{5}\right\} ; G=\left\{\left(b_{1}, e_{1}\right),\left(e_{1}, b_{3}\right),\left(b_{3}, e_{2}\right),\left(b_{2}, e_{2}\right), \ldots,\left(e_{5}, b_{9}\right),\left(e_{5}, b_{10}\right)\right\} ; l\left(e_{1}\right)=l\left(e_{3}\right)=b$, $l\left(e_{2}\right)=l\left(e_{4}\right)=$ tick, $l\left(e_{5}\right)=a$. We see that $|\cdot b| \leq 1 \wedge|b \bullet| \leq 1$, for all $b \in B$, and $\bullet \widetilde{T N}=$ $\left\{b_{1}, b_{2}\right\}$. Clearly, $e_{1} \prec e_{2} \prec e_{3} \prec e_{4} \prec e_{5}$. Moreover, $\left\{b_{1}, b_{2}\right\},\left\{b_{2}, b_{3}\right\}, \ldots,\left\{b_{9}, b_{10}\right\}$ are cuts in $\widetilde{T N}$. It is easy to check that $\tilde{\rho}=e_{1}, e_{2}, e_{3}, e_{4}, e_{5}$ is a linearization of $\widetilde{T N}$, because each event of $\widetilde{T N}$ appears in the sequence exactly once, and if $e_{i}<e_{j}$, then $i<j$, for all $1 \leq i, j \leq 5$. Define the downward closed sets $E_{i}=\cup_{1 \leq j \leq k} e_{j}$ and the sets $\operatorname{Cut}\left(E_{i}\right)=\left(\bullet T N \cup E_{i} \bullet\right) \backslash \bullet E_{i}=\left(\left\{b_{1}, b_{2}\right\} \cup\right.$ $\left.E_{i} \bullet\right) \backslash \cdot E_{i}$, for all $0 \leq i \leq 5$, as follows:

- $E_{0}=\varnothing, \operatorname{Cut}\left(E_{0}\right)=\left(\left\{b_{1}, b_{2}\right\} \cup \emptyset\right) \backslash \emptyset=\left\{b_{1}, b_{2}\right\} ;$

- $E_{1}=\left\{e_{1}\right\}, \operatorname{Cut}\left(E_{1}\right)=\left(\left\{b_{1}, b_{2}\right\} \cup\left\{b_{3}\right\}\right) \backslash\left\{b_{1}\right\}=\left\{b_{2}, b_{3}\right\}$

- $E_{2}=\left\{e_{1}, e_{2}\right\}, \operatorname{Cut}\left(E_{2}\right)=\left(\left\{b_{1}, b_{2}\right\} \cup\left\{b_{3}, b_{4}, b_{5}\right\}\right) \backslash\left\{b_{1}, b_{2}, b_{3}\right\}=\left\{b_{4}, b_{5}\right\}$;

- $E_{3}=\left\{e_{1}, e_{2}, e_{3}\right\}, \operatorname{Cut}\left(E_{3}\right)=\left(\left\{b_{1}, b_{2}\right\} \cup\left\{b_{3}, b_{4}, b_{5}, b_{6}\right\}\right) \backslash\left\{b_{1}, b_{2}, b_{3}, b_{5}\right\}=\left\{b_{4}, b_{6}\right\} ;$

- $E_{4}=\left\{e_{1}, e_{2}, e_{3}, e_{4}\right\}, \quad \operatorname{Cut}\left(E_{4}\right)=\left(\left\{b_{1}, b_{2}\right\} \cup\left\{b_{3}, b_{4}, b_{5}, b_{6}, b_{7}, b_{8}\right\}\right) \backslash\left\{b_{1}, b_{2}, b_{3}, b_{4}, b_{5}, b_{6}\right\}=$ 272 
$\left\{b_{7}, b_{8}\right\}$

- $E_{5}=\left\{e_{1}, e_{2}, e_{3}, e_{4}, e_{5}\right\}, \operatorname{Cut}\left(E_{5}\right)=B \backslash\left\{b_{1}, b_{2}, b_{3}, b_{4}, b_{5}, b_{6}, b_{7}, b_{8}\right\}=\left\{b_{9}, b_{10}\right\}$

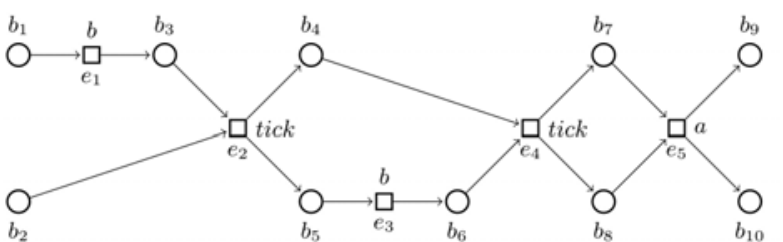

Fig. 2. A casual net $\widetilde{T N}$

Proposition 2. Any casual net $T N$ has a linearization $\rho=e_{1} \ldots e_{n}$

Proof. Take the maximal (w.r.t. set inclusion) set $\min (E) \subseteq E$ such that if $e \in \min (E)$, then $\neg\left(e^{\prime} \prec e\right)$, for all $e^{\prime} \in E$. After removing an event $e_{\min }$ from $E$, we get that $\min \left(E^{\prime}\right)=$ $\min (E) \backslash e_{\min }$ or $\min \left(E^{\prime}\right)=\left(\min (E) \backslash e_{\min }\right) \cup\left(e_{\min } \bullet\right)^{\bullet}$, where $E^{\prime}=E \backslash e_{\min }$. Construct a sequence $\rho=e_{1} \ldots e_{n}$ of events, by selecting a minimal event and removing it from $E$, at each step. By the construction, any event of $T N$ appears in the sequence exactly once and $e_{i} \prec e_{j} \Rightarrow i<j$, for all $1 \leq i, j \leq n$, due to $T N$ being a causal net.

The results of the below lemma will be useful to establish the relationships between "markings" (values of the function Cut) of a causal net $T N$ and markings of a time Petri net $\mathcal{T} \mathcal{N}$, and between linearizations of $T N$ and runs of $\mathcal{T N}$, when we construct partial order semantic for time Petri nets.

Lemma 2. Let $T N$ be a casual net and $\rho=e_{1} \ldots e_{n}$ its linearization. Then, it holds:

a) $\operatorname{Cut}\left(E_{k}\right)=\left(\operatorname{Cut}\left(E_{k-1}\right) \backslash \cdot e_{k}\right) \cup e_{k} \bullet$, for all $1 \leq k \leq n$;

b) $\cdot e_{k} \subseteq \operatorname{Cut}\left(E_{k-1}\right)$, for all $1 \leq k \leq n$;

c) if $\operatorname{Cut}\left(E_{k}\right) \neq \emptyset$, then $\operatorname{Cut}\left(E_{k}\right)$ is a cut of $T N$, for all $0 \leq k \leq n$.

\section{Proof. See Appendix.}

Next, we introduce a notion of a homomorphism from a causal net to a time Petri net, in order to define the concept of time processes of the time Petri net.

Definition 7. Let $\mathcal{T N}=\left(\left(P, T, F, M_{0}, L\right), D\right)$ be a TPN and $T N=(B, E, G, l)$ be a casual net. A homomorphism $^{l}$ from $T N$ to $\mathcal{T N}$ is a mapping $\varphi:(B \cup E) \rightarrow(P \cup T \cup\{\sqrt{ }\})$ such that it holds the following:

$-\varphi(B) \subseteq P$ and $\varphi(E) \subseteq(T \cup\{\sqrt{ }\})$;

- for all $e \in E$ such that $\varphi(e) \in T$,

$\circ$ the restriction of $\varphi$ to $\bullet e$ is a bijection between $\bullet e$ and $\bullet \varphi(e)$

○ the restriction of $\varphi$ to $e \bullet$ is a bijection between $e \bullet$ and $\varphi(e) \bullet$

- for all $e \in E$ such that $\varphi(e)=\sqrt{ }$,

${ }^{1}$ In fact, $\varphi$ is a homomorphism from $T N$ to $\mathcal{T N} \mathcal{N}^{\prime}=\left(\left(P, T, F^{\prime}, M_{0}, L^{\prime}\right), D\right)$, where $F^{\prime} \subseteq(P \times T \cup\{\sqrt{ }\}) \cup$ $(T \cup\{\sqrt{ }\} \times P)$ such that $F^{\prime}=F \cup\{(p, \sqrt{ }),(\sqrt{ }, p) \mid p \in P\}$, and $L^{\prime}: T \cup\{\sqrt{ }\} \rightarrow A c t \cup\{$ tick $\}$, such that $L^{\prime}(x)=\left\{\begin{array}{l}L(x), \text { if } x \in T \\ \text { tick, otherwise }\end{array}\right.$. In this case, we can see that $\varphi$ is a structure-preserving mapping. However, following the traditions of terms and definitions in the literature on TPNs, we omit this construction of $\mathcal{T} \mathcal{N}^{\prime}$. the restriction of $\varphi$ to $\bullet e$ and the restriction of $\varphi$ to $e \bullet$ are injections,

$\circ \bullet e$ and $e \bullet$ are cuts of $T N$ and $\varphi(\bullet e)=\varphi(e \bullet)$;

- the restriction of $\varphi$ to $\bullet T N$ is a bijection between $\bullet T N$ and $M_{0}$,

- $l(e)=\left\{\begin{array}{cc}L(\varphi(e)), & \text { if } \varphi(e) \in T, \\ \text { tick, } & \text { otherwise }\end{array}\right.$, for all $e \in E$

Then, the pair $\pi=(T N, \varphi)$ is called a time process of $\mathcal{T} \mathcal{N}$

Time processes $\pi=(T N, \varphi)=((B, E, G, l), \varphi)$ and $\pi^{\prime}=\left(T N^{\prime}, \varphi^{\prime}\right)=\left(\left(B^{\prime}, E^{\prime}, G^{\prime}, l^{\prime}\right), \varphi^{\prime}\right)$ of $\mathcal{T} \mathcal{N}$ are isomorphic (denoted $\pi \simeq \pi^{\prime}$ ) if there is an isomorphism $\gamma: T N \simeq T N^{\prime}$ such that $\varphi(x)=$ $\varphi^{\prime}(\gamma(x))$, for all $x \in B \cup E$

Example 7. Consider the TPN $\widetilde{\mathcal{N N}}$ depicted in Fig. 1, the casual net $\widetilde{T N}$ shown in Fig. 2, and a mapping $\varphi$ defined as follows: $\varphi\left(b_{1}\right)=\varphi\left(b_{9}\right)=p_{1}, \varphi\left(b_{2}\right)=\varphi\left(b_{5}\right)=\varphi\left(b_{10}\right)=p_{2}, \varphi\left(b_{3}\right)=$ $\varphi\left(b_{4}\right)=\varphi\left(b_{7}\right)=p_{3}, \varphi\left(b_{6}\right)=\varphi\left(b_{8}\right)=p_{4}, \varphi\left(e_{1}\right)=t_{1}, \varphi\left(e_{5}\right)=t_{2}, \varphi\left(e_{3}\right)=t_{3}, \varphi\left(e_{2}\right)=$

$\varphi\left(e_{4}\right)=\sqrt{ }$; Then, we have that $\varphi(B) \subseteq P, \varphi(E) \subseteq(T \cup\{\sqrt{ }\})$, and the restriction of $\varphi$ to $\bullet T N=$ $\left\{b_{1}, b_{2}\right\}$ is a bijection between $\left\{b_{1}, b_{2}\right\}$ and $M_{0}=\left\{p_{1}, p_{2}\right\}$. Moreover, it holds the following: for all $e \in E$ such that $\varphi(e) \in T$, the restriction of $\varphi$ to $\bullet \mathrm{e}(e \bullet)$ is a bijection between $\bullet \mathrm{e}(e \bullet)$ and - $\varphi(\mathrm{e})(\varphi(e) \bullet)$; and for all $e \in E$ such that $\varphi(e)=\sqrt{ }$, the restriction of $\varphi$ to $\bullet e(e \bullet)$ is an injection, $\bullet e$ and $e \bullet$ are cuts of $T N$ and $\varphi(\bullet e)=\varphi(e \bullet)$. For example, consider the events $e_{1}$ and $e_{2}$. We know that $\varphi\left(e_{1}\right)=t_{1}$ and $\varphi\left(e_{2}\right)=\sqrt{ }$. The restriction of $\varphi$ to $\bullet e_{1}=\left\{b_{1}\right\}\left(e_{1} \bullet=\left\{b_{3}\right\}\right)$ is a bijection between $\left\{b_{1}\right\}\left(\left\{b_{3}\right\}\right)$ and $\varphi\left(b_{1}\right)=\left\{p_{1}\right\}\left(\varphi\left(b_{3}\right)=\left\{p_{3}\right\}\right)$. Furthermore, the restriction of $\varphi$ to $\bullet e_{2}=$ $\left\{b_{2}, b_{3}\right\}\left(e_{2} \bullet=\left\{b_{4}, b_{5}\right\}\right)$ is an injection, $\left\{b_{2}, b_{3}\right\}$ and $\left\{b_{4}, b_{5}\right\}$ are cuts of $T N$ and $\varphi\left(\left\{b_{2}, b_{3}\right\}\right)=$

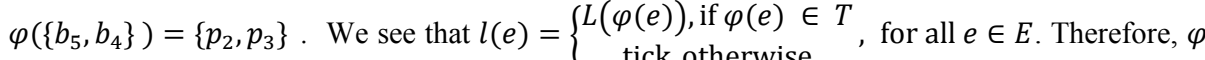
is the homomorphism from $\widetilde{T N}$ to $\widetilde{\mathcal{N N}}$, and, hence, $\pi=(\widetilde{T N}, \varphi)$ is a time process of $\widetilde{\mathcal{T N}}$.

For a time process $\pi=(T N, \varphi)$ of $\mathcal{T} \mathcal{N}$, we introduce the function Age that defines "age" of each condition $b$ of $T N$. More specifically, if $b \in \bullet T N$ is an input condition in $T N$, i.e. the place $\varphi(b)$ contains a token at the initial marking of $\mathcal{T} \mathcal{N}$, then the "age" of $b$ is equal to 0 . Also, if $b$ is an output condition of an event $e$ that corresponds to the firing of the transition $\varphi(e)$ of $\mathcal{T} \mathcal{N}$, i.e. the place $\varphi(b)$ of $\mathcal{T N}$ got a token immediately after the firing of $\varphi(e)$, then the "age" of $b$ is equal to 0 Otherwise, i.e. if $b$ is an output condition of an event $e$ that corresponds to time elapsing, then the "age" of $b$ is the increased by 1 "age" of the input condition $b$ ' of $e$, such that $\varphi(b)=\varphi(b$ '), i.e. $b$ and $b^{\prime}$ match in the same place of $\mathcal{T N}$.

$$
\operatorname{Age}(b)=\left\{\begin{array}{cc}
0, & \text { if } b \in \bullet T N \vee(b \in e \bullet, \varphi(e) \in T), \\
\text { Age }\left(b^{\prime}\right)+1, & \text { if } b \in e \bullet, \varphi(e)=\sqrt{ }, b^{\prime} \in \bullet e, \varphi\left(b^{\prime}\right)=\varphi(b) .
\end{array}\right.
$$

Notice that if $b \in e \bullet$ and $\varphi(e)=\sqrt{ }$, then there exists the only one condition $b^{\prime} \in \bullet e$ such that $\varphi\left(b^{\prime}\right)=\varphi(b)$, due to Definition 7. In this case, the definition of the function Age is correct. Informally speaking, the function Age matches each condition $b$ of $T N$ to the amount of time that has elapsed since the corresponding place $\varphi(b)$ of $\mathcal{T} \mathcal{N}$ got a token, when $\mathcal{T N}$ progresses.

For a co-set $B^{\prime}$ of conditions of $T N$ and a transition $t$ of $\mathcal{T N}$ such that $t$ is enabled at the marking $\varphi\left(B^{\prime}\right)$, determine the function Clock whose value is equal to the minimum "age" of the conditions from $B^{\prime}$, that correspond the input places of $t$.

$$
\operatorname{Clock}\left(B^{\prime}, t\right)=\left\{\begin{array}{cc}
\perp, & \text { if } \bullet t=\emptyset \\
\min \left\{\operatorname{Age}(b) \mid \varphi(b) \in \bullet t, b \in B^{\prime}\right\}, & \text { otherwise }
\end{array}\right.
$$


Informally speaking the function Clock matches $B^{\prime}$ and $t$ to the amount of time that has elapsed since a token from the marking $\varphi\left(B^{\prime}\right)$ appeared in the last input place of the transition $t$, i.e. since the transition $t$ became enabled at $\varphi\left(B^{\prime}\right)$. Later, we will establish a correspondence between the function Clock and the dynamic timing function $I$, when the TPN progresses.

We are now ready to introduce the concept of an admissible (correct) time process of $\mathcal{T N}$

Definition 8. Let $\mathcal{T} \mathcal{N}$ be a time Petri net. A time process $\pi=(T N, \varphi)$ of $\mathcal{T} \mathcal{N}$ is admissible iff for all $e \in E$ it holds:

$\varphi(e) \in T \Rightarrow \operatorname{Eft}(\varphi(e)) \leq \operatorname{Clock}(\bullet e, \varphi(e)) \leq \operatorname{Lft}(\varphi(e))$.

Example 8. Verify that the time process $\pi$ from Example 7 of $\widetilde{\mathcal{N N}}$ shown in Fig. 1 is admissible. By definitions, we have:

- $\operatorname{Clock}\left(\cdot e_{1}, \varphi\left(e_{1}\right)\right)=\operatorname{Age}\left(b_{1}\right)=0$

- $\operatorname{Clock}\left(\cdot e_{3}, \varphi\left(e_{3}\right)\right)=\operatorname{Age}\left(b_{5}\right)=\operatorname{Age}\left(b_{2}\right)=0+1=1$;

$-\operatorname{Clock}\left(\bullet e_{5}, \varphi\left(e_{5}\right)\right)=\min \left\{\operatorname{Age}\left(b_{7}\right), \operatorname{Age}\left(b_{8}\right)\right\}=\min \left\{\operatorname{Age}\left(b_{4}\right)+1, \operatorname{Age}\left(b_{6}\right)=0+1\right\}=$ $\min \left\{\left(\operatorname{Age}\left(b_{3}\right)=0+1\right)+1,1\right\}=1$.

Then, we obtain:

- $\operatorname{Eft}\left(t_{1}\right)=0 \leq \operatorname{Clock}\left(\cdot e_{1}, \varphi\left(e_{1}\right)=t_{1}\right) \leq 1=\operatorname{Lft}\left(\varphi\left(e_{1}\right)\right)$

- $\operatorname{Eft}\left(t_{3}\right)=0 \leq \operatorname{Clock}\left(\cdot e_{3}, \varphi\left(e_{3}\right)=t_{3}\right) \leq 2=\operatorname{Lft}\left(t_{3}\right)$,

- $\operatorname{Eft}\left(t_{2}\right)=1 \leq \operatorname{Clock}\left(\cdot e_{5}, \varphi\left(e_{5}\right)=t_{2}\right) \leq 3=\operatorname{Lft}\left(t_{2}\right)$.

So, $\pi$ is an admissible time process of $\widetilde{\mathcal{T N}}$.

\section{Relating Runs and Time Processes of $\mathcal{T N}$}

In this section, relationships between runs and linearizations (computations) of admissible time processes are investigated, in the context of time Petri nets. For this purpose, we define a mapping $F S$ from a linearization $\rho=e_{1} \ldots e_{n}$ of a time process $\pi=(T N, \varphi)$ of the TPN $\mathcal{T} \mathcal{N}$ to the sequence of the form: $F S(\rho)=\varphi\left(e_{1}\right) \ldots \varphi\left(e_{n}\right)$. Here, $T N$ is a causal net and $\varphi$ is a homomorphism from $T N$ to $\mathcal{T} \mathcal{N}$.

First, we prove that if $F S$ maps a prefix $\rho_{i}(0 \leq i \leq n)$ of the linearization $\rho$ to the run of $\mathcal{T N}$ and $\left(M_{i}, I_{i}\right)$ is the state reachable by the run, then $\varphi$ maps the value of the function Cut of this prefix to the marking $M_{i}$. Moreover, for any transition $t$ enabled at $M_{i}$, the value of the dynamic timing function $I_{i}(t)$ is equal to $\operatorname{Clock}\left(\operatorname{Cut}\left(E_{i}\right), t\right)$, where $E_{i}$ is the set of events from $\rho_{i}$.

Lemma 3. Let $\pi=(T N, \varphi)$ be a time process of the TPN $\mathcal{T N}$ and $\rho=e_{1} \ldots e_{n}$ be a linearization of $T N$. If $F S\left(\rho_{i}\right)=\varphi\left(e_{1}\right) \ldots \varphi\left(e_{i}\right)$ is the run of $\mathcal{T N}$ from $\left(M_{0}, I_{0}\right)$ to $\left(M_{i}, I_{i}\right)$ for some $0 \leq i \leq$ $n$, then it holds:

a) the restriction of $\varphi$ to $\operatorname{Cut}\left(E_{i}\right)$ is a bijection between $\operatorname{Cut}\left(E_{i}\right)$ and $M_{i}$;

b) $\operatorname{Clock}\left(\operatorname{Cut}\left(E_{i}\right), t\right)=I_{i}(t)$, for all $t \in \operatorname{En}\left(M_{i}\right)$;

c) if $i<n$ and $\varphi\left(e_{i+1}\right) \in \operatorname{En}\left(M_{i}\right)$, then $\operatorname{Clock}\left(\bullet e_{i+1}, \varphi\left(e_{i+1}\right)=I_{i}\left(\varphi\left(e_{i+1}\right)\right)\right.$.

Proof. See Appendix.

We are now ready to establish an important property of the $F S$ mapping - any linearization of a time process of the TPN is mapped to its runs.

Theorem 3. Given an admissible time process $\pi=(T N, \varphi)$ of $\mathcal{T} \mathcal{N}$ and a linearization $\rho=e_{1} \ldots e_{n}$ of $T N, F S(\rho)$ is a run of $\mathcal{T N}$.
Proof. We shall prove by induction on $0 \leq i \leq n$ that $F S\left(\rho_{i}\right)$ is a run of $\mathcal{T N}$.

$i=0$. Then, $F S\left(\rho_{0}\right)$ is the empty run.

$i>0$. By the induction hypothesis, $F S\left(\rho_{i-1}\right)$ is a run of $\mathcal{T N}$. If $\varphi\left(e_{i}\right)=\sqrt{ }$, then $F S\left(\rho_{i}\right)$ is a run of $\mathcal{T N}$, due to Definition 2. Assume $\varphi\left(e_{i}\right) \in T$. By Lemma 2(b), we have $\varphi\left(\bullet e_{i}\right) \subseteq \varphi\left(\operatorname{Cut}\left(E_{i-1}\right)\right)$. As the restriction of $\varphi$ to $\bullet e$ is a bijection between $\bullet e$ and $\bullet \varphi(e)$, we obtain $\bullet \varphi\left(e_{i}\right) \subseteq \varphi\left(\operatorname{Cut}\left(E_{i-1}\right)\right)$. Thus, we get $\varphi\left(e_{i}\right) \in E n\left(M_{i-1}\right)$, due to Lemma 3(a). Thanks to Lemma 3(c), we have that $I_{i-1}\left(\varphi\left(e_{i}\right)\right)=\operatorname{Clock}\left(\bullet e_{i}, \varphi\left(e_{i}\right)\right)$. Then, it holds that $\operatorname{Eft}\left(\varphi\left(e_{i}\right)\right) \leq I_{i-1}\left(\varphi\left(e_{i}\right)\right) \leq \operatorname{Lft}\left(\varphi\left(e_{i}\right)\right)$, by Definition 8. Therefore, $\varphi\left(e_{i}\right)$ can fire from $\left(M_{i-1}, I_{i-1}\right)$, i.e. $F S\left(\rho_{i}\right)$ is a run of $\mathcal{T} \mathcal{N}$.

Next, we show that the mapping $F S$ is a surjection, i.e. for an arbitrary run $\sigma$ of $\mathcal{T} \mathcal{N}$, there is exists an admissible time process $\pi^{*}=\left(T N^{*}, \varphi^{*}\right)$ of $\mathcal{T} \mathcal{N}$ and a linearization $\rho^{*}$ of $T N^{*}$ such that $F S\left(\rho^{*}\right)=$ $\sigma$. The following definition provides constructions of $\pi^{*}$ and $\rho^{*}$.

Definition 9. Let $\mathcal{T} \mathcal{N}=\left(\left(P, T, F, M_{0}, L\right), D\right)$ be a time Petri net and $\sigma=\overline{t_{1}} \ldots \overline{t_{n}} \in\left(T \cup\{\sqrt{\}})^{n}\right.$ be a run of $\mathcal{T} \mathcal{N}$.

We construct a finite sequence of tuples $\left(E_{i}, B_{i}, G_{i}, C_{i}\right)$ by induction on $0 \leq i \leq n$.

$i=0$. Then, set:

$-E_{0}=\varnothing$

- $B_{0}=\left\{b_{0, p} \mid p \in M_{0}\right\}$

- $G_{0}=\varnothing$

$-C_{0}=B_{0}$

$i>0$. Assume that $\left(E_{i-1}, B_{i-1}, G_{i-1}, C_{i-1}\right)$ is already constructed. Then, set:

- $E_{i}=E_{i-1} \cup\left\{e_{i}\right\}$

- $B_{i}=B_{i-1} \cup\left\{\begin{array}{cc}\left\{b_{i, p} \mid b_{j, p} \in C_{i-1}\right\}, & \text { if } \overline{t_{i}}=\sqrt{ }, \\ \left\{b_{i, p} \mid p \in \overline{t_{i}} \bullet\right\}, & \text { otherwise }\end{array}\right.$

- $G_{i}=G_{i-1} \cup\left\{\left(e_{i}, b_{i, p}\right) \mid b_{i, p} \in B_{i}\right\} \cup\left\{\begin{array}{cl}\left\{\left(b_{j, p}, e_{i}\right) \mid b_{j, p} \in C_{i-1}\right\}, & \text { if } \overline{t_{i}}=\sqrt{ }, \\ \left\{\left(b_{j, p}, e_{i}\right) \mid b_{j, p} \in C_{i-1}, p \in \bullet \overline{t_{i}}\right\}, & \text { otherwise }\end{array}\right.$

- $C_{i}=\left(C_{i-1} \backslash \bullet e_{i}\right) \cup e_{i} \bullet$

Define $\pi^{*}=\left(T N^{*}, \varphi^{*}\right)=\left(\left(B, E, G, l^{*}\right), \varphi^{*}\right)$ as follows:

- $B=B_{n}, E=E_{n}, G=G_{n}$

- $\varphi^{*}\left(e_{i}\right)=\overline{t_{i}}$, for all $e_{i} \in E$, and $\varphi^{*}\left(b_{i, p}\right)=p$, for all $b_{i, p} \in B$;

- $l^{*}(e)=\left\{\begin{array}{cc}\text { tick, } & \text { if } \varphi^{*}(e)=\sqrt{ }, \\ L\left(\varphi^{*}(e)\right), & \text { otherwise }\end{array}\right.$, for all $e \in E$.

Determine $\rho^{*}=e_{1} \ldots e_{n}$.

Example 9. Consider the time Petri net $\widetilde{\mathcal{T N}}$ depicted in Fig. 1 and its run $\sigma=t_{1} \sqrt{t_{3}} \sqrt{t_{2}}$. We construct the sequence of the following tuples $\left(E_{i}, B_{i}, G_{i}, C_{i}\right)$, with $0 \leq i \leq 5$.

- $i=0$. Set $E_{0}=\emptyset ; C_{0}=B_{0}=\left\{b_{0, p} \mid p \in M_{0}=\left\{p_{1}, p_{2}\right\}\right\}=\left\{b_{0, p_{1}}, b_{0, p_{2}}\right\} ; G_{0}=\emptyset$.

- $i=1$. Set $E_{1}=E_{0} \cup\left\{e_{1}\right\} ; \quad B_{1}=B_{0} \cup\left\{b_{1, p} \mid p \in t_{1} \bullet=\left\{p_{3}\right\}\right\}=\left\{b_{1, p_{3}}\right\} ; \quad G_{1}=G_{0} \cup$ $\left\{\left(e_{1}, b_{1, p}\right) \mid b_{1, p} \in B_{1}\right\} \cup\left\{\left(b_{j, p}, e_{1}\right) \mid b_{j, p} \in C_{0}, p \in \bullet t_{1}=\left\{p_{1}\right\}\right\}=G_{0} \cup$

$\left\{\left(e_{1}, b_{1, p_{3}}\right),\left(b_{0, p_{1}}, e_{1}\right)\right\} ; C_{1}=\left(C_{0} \backslash \bullet e_{1}\right) \cup e_{1} \bullet=\left\{b_{0, p_{2}}, b_{1, p_{3}}\right\}$.

- $i=2$. Set $\quad E_{2}=E_{1} \cup\left\{e_{2}\right\} ; \quad B_{2}=B_{1} \cup\left\{b_{2, p} \mid b_{j, p} \in C_{1}\right\}=\left\{b_{2, p_{2}}, b_{2, p_{3}}\right\} ; \quad G_{2}=G_{1} \cup$ $\left\{\left(e_{2}, b_{2, p}\right) \mid b_{2, p} \in B_{2}\right\} \cup\left\{\left(b_{j, p}, e_{2}\right) \mid b_{j, p} \in C_{1}\right\}=G_{1} \cup$

$\left\{\left(e_{2}, b_{2, p_{3}}\right),\left(e_{2}, b_{2, p_{2}}\right),\left(b_{1, p_{3}}, e_{2}\right),\left(b_{0, p_{2}}, e_{2}\right)\right\} ; C_{2}=\left(C_{1} \backslash \bullet e_{2}\right) \cup e_{2} \bullet=\left\{b_{2, p_{3}}, b_{2, p_{2}}\right\}$.

- $i=3$. Set $E_{3}=E_{2} \cup\left\{e_{3}\right\} ; \quad B_{3}=B_{2} \cup\left\{b_{3, p} \mid p \in t_{3} \bullet=\left\{p_{4}\right\}\right\}=\left\{b_{3, p_{4}}\right\} ; \quad G_{3}=G_{2} \cup$ 
$\left\{\left(e_{3}, b_{3, p}\right) \mid b_{3, p} \in B_{3}\right\} \cup\left\{\left(b_{j, p}, e_{3}\right) \mid b_{j, p} \in C_{2}, p \in \bullet t_{3}=\left\{p_{2}\right\}\right\}=G_{3} \cup$

$\left\{\left(e_{3}, b_{3, p_{4}}\right),\left(b_{2, p_{2}}, e_{3}\right)\right\} ; C_{3}=\left(C_{2} \backslash \bullet e_{3}\right) \cup e_{3} \bullet=\left\{b_{2, p_{3}}, b_{3, p_{4}}\right\}$

- $i=4$. Set $\quad E_{4}=E_{3} \cup\left\{e_{4}\right\} ; \quad B_{4}=B_{3} \cup\left\{b_{4, p} \mid b_{j, p} \in C_{3}\right\}=\left\{b_{4, p_{3}}, b_{4, p_{4}}\right\} ; \quad G_{4}=G_{3} \cup$ $\left\{\left(e_{4}, b_{4, p}\right) \mid b_{4, p} \in B_{4}\right\} \cup\left\{\left(b_{j, p}, e_{4}\right) \mid b_{j, p} \in C_{3}\right\}=G_{3} \cup$

$\left\{\left(e_{4}, b_{4, p_{3}}\right),\left(e_{4}, b_{4, p_{4}}\right),\left(b_{2, p_{3}}, e_{4}\right),\left(b_{3, p_{4}}, e_{4}\right)\right\} ; C_{4}=\left(C_{3} \backslash \bullet e_{4}\right) \cup e_{4} \bullet=\left\{b_{4, p_{3}}, b_{4, p_{4}}\right\}$

- $i=5$. Set $E_{5}=E_{4} \cup\left\{e_{5}\right\} ; B_{5}=B_{4} \cup\left\{b_{5, p} \mid p \in t_{2} \bullet=\left\{p_{1}, p_{2}\right\}\right\}=\left\{b_{5, p_{1}}, b_{5, p_{2}}\right\} ; G_{5}=G_{4} \cup$ $\left\{\left(e_{5}, b_{5, p}\right) \mid b_{5, p} \in B_{5}\right\} \cup\left\{\left(b_{j, p}, e_{5}\right) \mid b_{j, p} \in C_{4}, p \in \bullet t_{2}=\left\{p_{3}, p_{4}\right\}\right\}=G_{4} \cup$

$\left\{\left(e_{5}, b_{5, p_{1}}\right),\left(e_{5}, b_{5, p_{2}}\right),\left(b_{4, p_{3}}, e_{5}\right),\left(b_{4, p_{4}}, e_{5}\right)\right\} ; C_{5}=\left(C_{4} \backslash \bullet e_{5}\right) \cup e_{5} \bullet=\left\{b_{5, p_{1}}, b_{5, p_{2}}\right\}$.

Determine the following: $\varphi^{*}\left(e_{i}\right)=\overline{t_{i}}$, for all $e_{i} \in E_{5}, \varphi^{*}\left(b_{j, p}\right)=p$, for all $b_{j, p} \in B_{5}, l^{*}\left(e_{i}\right)=$ $\left\{\right.$ tick, if $\varphi^{*}\left(e_{i}\right)=\sqrt{ }$,

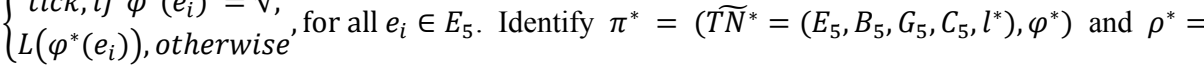
$e_{1} \ldots e_{n}$. Notice that $\widetilde{T N}^{*}$ and $\widetilde{T N}$ from Example 6 are equal up to renaming their conditions. $\square$

The following lemmas demonstrate important properties of the constructions from Definition 9.

Lemma 4.

a) $T N^{*}$ is a casual net;

b) $\rho^{*}=e_{1} \ldots e_{n}$ is a linearization of $T N^{*}$;

c) $C_{i}=\operatorname{Cut}\left(E_{i}\right)$, for all $0 \leq i \leq n$.

Proof. See Appendix.

Lemma 5. The mapping $\varphi^{*}$ is a homomorphism from $T N^{*}$ to $\mathcal{T} \mathcal{N}$.

Proof. See Appendix.

We are now ready to establish that $F S$ is a surjective mapping.

Theorem 4. Given a run $\sigma$ of a time Petri net $\mathcal{T N}$, there exists an admissible time process $\pi^{*}=$ $\left(T N^{*}, \varphi^{*}\right)$ of $\mathcal{T N}$ and a linearization $\rho^{*}=e_{1} \ldots e_{n}$ of $T N^{*}$ such that $\sigma=F S\left(\rho^{*}\right)=$ $\varphi^{*}\left(e_{1}\right) \ldots \varphi^{*}\left(e_{n}\right)$.

Proof. Consider the construction of $\pi^{*}$ from Definition 9. According to Lemma 4(a) and Lemma 5 , $\pi^{*}$ is a time process of $\mathcal{T N}$. Take an arbitrary $1 \leq i \leq n$ such that $\varphi^{*}\left(e_{i}\right) \in T$. Then, $\operatorname{Clock}\left(\bullet e_{i}, \varphi^{*}\left(e_{i}\right)\right)=I_{i-1}\left(\varphi^{*}\left(e_{i}\right)\right)$, by Lemma 3(c). Due to $\sigma$ being a run of $\mathcal{T} \mathcal{N}$, we obtain that $\operatorname{Eft}\left(\varphi^{*}\left(e_{i}\right)\right) \leq \operatorname{Clock}\left(\cdot e_{i}, \varphi^{*}\left(e_{i}\right)\right) \leq \operatorname{Lft}\left(\varphi^{*}\left(e_{i}\right)\right)$. Hence, $\pi^{*}$ is an admissible time process of $\mathcal{T N}$. Thanks to Lemma 4(b), $\rho^{*}=e_{1} \ldots e_{n}$ is a linearization of $T N^{*}$. By the construction of $\varphi^{*}$, we get that $F S\left(\rho^{*}\right)=\sigma$

$\square$

The following theorem shows that $F S$ is an injection, i.e. the constructed in Definition 9 time process $\pi^{*}=\left(T N^{*}, \varphi^{*}\right)$ is unique up to isomorphism.

Theorem 5. Let $\sigma$ be a run of $\mathcal{T} \mathcal{N}$. The time process $\pi=(T N, \varphi)$ of $\mathcal{T} \mathcal{N}$ with linearization $\rho=$ $e_{1} \ldots e_{n}$ of $T N$, such that $\sigma=F S(\rho)=\varphi\left(e_{1}\right) \ldots \varphi\left(e_{n}\right)$ is unique up to isomorphism.

Proof. Take arbitrary time process $\pi^{\prime}=\left(T N^{\prime}=\left(B^{\prime}, E^{\prime}, G^{\prime}, l^{\prime}\right), \varphi^{\prime}\right)$ of $\mathcal{T} \mathcal{N}$ and linearization $\rho^{\prime}=$ $e^{\prime}{ }_{1} \ldots e_{n}^{\prime}(n \geq 0)$ of $T N^{\prime}$ such that $F S\left(\rho^{\prime}\right)=\sigma$. Then, $E^{\prime}=\left\{e^{\prime}, \ldots, e^{\prime}{ }_{n}\right\}$, by the definition of the linearization.

Moreover, $B^{\prime}=\bullet T N^{\prime} \oplus e^{\prime}{ }_{1} \bullet \oplus \ldots \oplus e^{\prime}{ }_{n} \cdot$, due to $T N^{\prime}$ being an acyclic net. Set $B_{0}^{\prime}=\bullet T N^{\prime}$ and $B_{i}^{\prime}=B_{i-1}^{\prime} \cup e_{i}^{\prime} \cdot$, for $1 \leq i \leq n$. By Definition 7 , the restriction of $\varphi^{\prime}$ to $\bullet T N^{\prime}$ is a bijection between
- $T N^{\prime}$ and $M_{0}$. Then, w.l.o.g. assume $B_{0}^{\prime}=\left\{b_{0, p}^{\prime} \mid p \in M_{0}\right\}$, with $\varphi^{\prime}\left(b_{0, p}^{\prime}\right)=p$. Take an arbitrary $1 \leq i \leq n$. Suppose $\varphi^{\prime}\left(e^{\prime}{ }_{i}\right) \in T$. Then, due to the restriction of $\varphi^{\prime}$ to $e^{\prime}{ }_{i} \bullet$ being a bijection between $e^{\prime}{ }_{i}^{\bullet}$ and $\varphi^{\prime}\left(e^{\prime}{ }_{i}\right) \bullet$, w.l.o.g. assume $e^{\prime}{ }_{i} \bullet=\left\{b_{i, p}^{\prime} \mid p \in \varphi^{\prime}\left(e^{\prime}{ }_{i}\right) \bullet\right\}$. If $\varphi^{\prime}\left(e^{\prime}{ }_{i}\right)=\sqrt{ }$, then $e^{\prime}{ }_{i} \bullet$ is a cut, i.e. $e_{i}^{\prime} \bullet \neq \varnothing$, thanks to Definition 7. In addition, we have that $e^{\prime} \cdot \subseteq \operatorname{Cut}\left(E^{\prime}{ }_{i}\right)$, by Lemma 2(a), and $\operatorname{Cut}\left(E^{\prime}{ }_{i}\right)$ is cut, by Lemma 2(c), i.e. $e_{i}^{\prime} \bullet=\operatorname{Cut}\left(E^{\prime}{ }_{i}\right)$. Thanks to Definition 2, it holds that $M_{i}=$ $M_{i-1}$. Hence, the restriction of $\varphi^{\prime}$ to $e_{i}{ }^{\prime} \bullet$ is a bijection between $e_{i}^{\prime} \bullet$ and $\left.\varphi^{\prime}\left(\operatorname{Cut}_{\left(E^{\prime}\right.}^{\prime}{ }_{i-1}\right)\right)$, according to Lemma 3(a). W.1.o.g. suppose $e_{i}^{\prime} \bullet=\left\{b^{\prime}{ }_{i, p} \mid p \in \varphi^{\prime}\left(\operatorname{Cut}\left(E^{\prime}{ }_{i-1}\right)\right)\right\}$. Thus, for all $1 \leq i \leq n$, we obtain the following:

- $B_{i}^{\prime}=B_{i-1}^{\prime} \cup\left\{\begin{array}{cc}e_{i}^{\prime} \bullet=\left\{b^{\prime}{ }_{i, p} \mid p \in \varphi^{\prime}\left(\operatorname{Cut}\left(E_{i-1}^{\prime}\right)\right)\right\}, & \text { if } \varphi^{\prime}\left(e_{i}^{\prime}\right)=\sqrt{ }, \\ e_{i}^{\prime} \cdot=\left\{b_{i, p}^{\prime} \mid p \in \varphi^{\prime}\left(e_{i}^{\prime}\right) \cdot\right\}, & \text { otherwise }\end{array} ;\right.$

- $\varphi^{\prime}\left(b_{i, p}^{\prime}\right)=p$, for all $b_{i, p}^{\prime} \in B_{i}^{\prime}$.

Compare the time process $\pi^{\prime}$ of $\mathcal{T} \mathcal{N}$ and the time process $\pi^{*}=\left(\left(B, E, G, l^{*}\right), \varphi^{*}\right)$ of $\mathcal{T} \mathcal{N}$ of $T N^{*}$ (from Definition 9). Clearly, $E^{\prime}$ and $E$ have the same cardinality. Due to $F S\left(\rho^{\prime}\right)=F S\left(\rho^{*}\right)$, we obtain that $\varphi^{\prime}\left(e_{i}^{\prime}\right)=\varphi^{*}\left(e_{i}\right)$, for all $1 \leq i \leq n$. According Lemma 3(a) and Lemma 4(c), it holds that $\varphi^{\prime}\left(\operatorname{Cut}\left(E_{i-1}\right)\right)=M_{i-1}=\varphi^{*}\left(\operatorname{Cut}\left(E_{i-1}\right)=C_{i-1}\right)$, for all $1 \leq i \leq n$. Hence, $B_{i}$ and $B_{i}^{\prime}$ have the same cardinality, for all $0 \leq i \leq n$.

Thanks to the definitions of $E^{\prime}\left(E^{*}\right)$ and $B^{\prime}\left(B^{*}\right)$, we can construct a bijective mapping $\gamma$ : $\left(E^{\prime} \cup B^{\prime}\right) \rightarrow(E \cup B)$, with $\gamma\left(e_{i}^{\prime}\right)=e_{i}$, for all $e_{i}^{\prime} \in E^{\prime}$, and $\gamma\left(b_{j, p}^{\prime}\right)=b_{j, p}$, for all $b_{j, p}^{\prime} \in B^{\prime}$ such that $\gamma\left(B^{\prime}\right)=B$ and $\gamma\left(E^{\prime}\right)=E$. Clearly, $\varphi^{\prime}(x)=\varphi^{*}(\gamma(x))$, for all $x \in B^{\prime} \cup E^{\prime}$, and hence, $l^{\prime}\left(e_{i}^{\prime}\right)=l^{*}\left(\gamma\left(e_{i}^{\prime}\right)\right)$, for all $e_{i}^{\prime} \in E^{\prime}$. It remains to show that $G^{\prime}$ is isomorphic to $G$. Take an arbitrary $1 \leq i \leq n$. Due to the definitions of $B_{i}$ and $B^{\prime}{ }_{i}$, we have that $\left(e_{i}^{\prime}, b_{j, p}^{\prime}\right) \in G^{\prime} \Leftrightarrow b_{j, p}^{\prime} \in B^{\prime}{ }_{i} \Leftrightarrow$ $b_{j, p} \in B_{i} \Leftrightarrow\left(e_{i}, b_{j, p}\right) \in G$. Check that $\left(b_{j, p}^{\prime}, e_{i}^{\prime}\right) \in G^{\prime} \Leftrightarrow\left(b_{j, p}, e_{i}\right) \in G$.

Claim. $b_{j, p} \in \operatorname{Cut}\left(E_{i-1}\right) \Leftrightarrow b_{j, p}^{\prime} \in \operatorname{Cut}\left(E^{\prime}{ }_{i-1}\right)$.

Proof. We prove the case with $b_{j, p} \in \operatorname{Cut}\left(E_{i-1}\right)$ (the case with $b_{j, p}^{\prime} \in \operatorname{Cut}\left(E^{\prime}{ }_{i-1}\right)$ is symmetric). Then, there exists $b^{\prime}{ }^{\prime}, p \in \operatorname{Cut}\left(E^{\prime}{ }_{i-1}\right)$, according to Lemma 3(a). Suppose a contrary, i.e. $j^{\prime} \neq j$. W.l.o.g. assume $j<j^{\prime}<i$. As $\gamma: B^{\prime} \rightarrow B$ is bijection, there exists $b_{j, p}=\gamma\left(b^{\prime}{ }_{j \prime, p}\right)$. Due to Definition 9, we have that $b_{j, p} \in \bullet T N$, if $j=0$, or $b_{j, p} \in e_{j} \bullet \subseteq E_{j} \bullet$, if $j>0$, and $b_{j, p} \in e_{j} \bullet$, because $j^{\prime}>j$. Then, $b_{j^{\prime}, p} \in \operatorname{Cut}\left(E_{j \prime}\right)$, by Lemma 2(a). However, $b_{j, p} \notin \operatorname{Cut}\left(E_{j \prime}\right)$, thanks to Lemma 3(a). Since $E_{j} \bullet \subseteq E_{j \prime} \bullet$, we get that $b_{j, p} \in \bullet T N \cup E_{j \prime} \bullet$. Hence, $b_{j, p} \in \bullet E_{j \prime}$, due to the definition of $\operatorname{Cut}\left(E_{j \prime}\right)$. This implies that $b_{j, p} \in \bullet E_{i-1}$, contradicting $b_{j, p} \in \operatorname{Cut}\left(E_{i-1}\right)$

According to Lemma 2(b), we have $\bullet e_{i}^{\prime} \subseteq \operatorname{Cut}\left(E_{i-1}^{\prime}\right)$. Assume that $\varphi^{\prime}\left(e_{i}^{\prime}\right)=\sqrt{ }$. Then, $\bullet e_{i}^{\prime}$ is a cut i.e $\bullet e_{i}^{\prime} \neq \varnothing$, due to Definition 7. Moreover, we have that $\operatorname{Cut}\left(E^{\prime}{ }_{i-1}\right)$ is cut, by Lemma 2(c), i.e. $\bullet e_{i}^{\prime}=$ $\operatorname{Cut}\left(E^{\prime}{ }_{i-1}\right)$. Therefore, $\quad\left(b_{j, p}^{\prime}, e_{i}^{\prime}\right) \in G^{\prime} \Leftrightarrow b_{j, p}^{\prime} \in \operatorname{Cut}\left(E^{\prime}{ }_{i-1}\right) \Leftrightarrow b_{j, p} \in \operatorname{Cut}\left(E_{i-1}\right) \Leftrightarrow$ $\left(b_{j, p}, e_{i}\right) \in G$, thanks to Claim. Assume $\varphi^{\prime}\left(e_{i}^{\prime}\right) \in T$. Then, it holds that $\bullet e_{i}^{\prime}=\left\{b_{j, p}^{\prime} \mid p \in \bullet \varphi^{\prime}\left(e_{i}^{\prime}\right) \wedge\right.$ $\left.b_{j, p}^{\prime} \in \operatorname{Cut}\left(E_{i-1}^{\prime}\right)\right\}$, due to the restriction of $\varphi^{\prime}$ to $\bullet e_{i}^{\prime}$ being a bijection between $\bullet e_{i}^{\prime}$ and $\bullet \varphi^{\prime}\left(e_{i}^{\prime}\right)$. By virtue of Claim, we get that $\left(b_{j, p}^{\prime}, e_{i}^{\prime}\right) \in G^{\prime} \Leftrightarrow p \in \bullet \varphi^{\prime}\left(e_{i}^{\prime}\right) \wedge b_{j, p}^{\prime} \in \operatorname{Cut}\left(E_{i-1}^{\prime}\right) \Leftrightarrow p \in$ - $\varphi^{*}\left(e_{i}\right) \wedge b_{j, p} \in \operatorname{Cut}\left(E_{i-1}\right) \Leftrightarrow\left(b_{j, p}, e_{i}\right) \in G$.

Therefore, we obtain that $\gamma: \pi^{\prime} \simeq \pi^{*}$.

Thus, we have demonstrated that $F S$ is a bijective mapping between linearizations of time processes and runs from the initial state, in the context of the TPN $\mathcal{T N}$. 


\section{Conclusion}

In this paper, we have introduced and studied partial order semantics for TPNs with weak time elapsing and intermediate memory policies. First, we have developed a state space discretization technique for the TPN, i.e. we have shown that any of its run with real-value time elapsings can be represented as that with the same untimed part and with only unit time elapsings. This allows us to transform time elapsings into the structure of a causal net with tick-events. Second, partial order semantics of the TPN has been proposed in the terms of time causal processes which consist of causal nets and their homomorphism into the TPN. Partial order semantics is useful for taking into account the processes' timing behavior in addition to their degrees of relative concurrency. Also, in the context of the TPN, a bijective mapping has been proved to exist between interleaving runs and computations (linearizations) of time causal processes, demonstrating that the partial order semantics is correct w.r.t. the interleaving that.

As for future work, we plan to extend the results obtained to atomic memory and back in time policies. As well, we believe that partial order semantics developed here allows us to elaborate and investigate behavioral equivalences of TPNs with weak semantics, in interleaving - partial order dichotomy.

\section{References}

1. M. Boyer and O. H. Roux. On the compared expressiveness of arc, place and transition time Petri nets. Fundamenta Informaticae, vol. 88, no. 3, 2008, pp. 225-249.

2. B. Bérard, F. Cassez, S. Haddad, D. Lime, and O. H. Roux. Comparison of different semantics for time Petri nets. Lecture Notes in Computer Science, vol. 3707, 2005, pp. 293-307.

3. P.-A. Reynier and A. Sangnier. Weak time Petri nets strike back! Lecture Notes in Computer Science, vol. 5710,2009 , pp. $557-571$.

4. L. Popova-Zeugmann. Time Petri nets. Springer, 2013, pp. 31-137.

5. T. Aura and J. Lilius. A causal semantics for time Petri nets. Theoretical Computer Science, vol. 243, no. 1-2, 2000, pp. 409-447.

6. H. Fleischhack and C. Stehno. Computing a finite prefix of a time Petri net. Lecture Notes in Computer Science, vol. 2360, 2002, pp. 163-181

7. T. Chatain and C. Jard. Back in time Petri nets. Lecture Notes in Computer Science, vol. 8053, 2013, pp.

91-105.

\section{Appendix}

Proof of Lemma 1. Let $\beta$ is a solution of $B_{\omega}$. We shall prove, that $\left(M_{0}, I_{0}\right) \stackrel{\left[\omega_{i}\right]_{\beta}}{\longrightarrow}\left(M_{\omega_{i}},\left[I_{\omega_{i}}^{\prime}\right]_{\beta}\right)$, for all $0 \leq i \leq k$, by induction on $i$.

$i=0$. Due to Definition 4, we get: $\omega_{0}=x_{0} ; M_{\omega_{0}}=M_{0} ; \forall t \in E n\left(M_{\omega_{0}}\right): I_{\omega_{0}}^{\prime}(t)=x_{0}$. Hence, $\left(M_{0}, I_{0}\right) \stackrel{\left[\omega_{0}\right]_{\beta}}{\longrightarrow}\left(M_{\omega_{0}},\left[I_{\omega_{0}}^{\prime}\right]_{\beta}\right)$, thanks to Definition 2.

$i>0$. By the induction hypothesis, we have that $\left(M_{0}, I_{0}\right) \stackrel{\left[\omega_{i-1}\right]_{\beta}}{\rightarrow}\left(M_{\omega_{i-1}},\left[I_{\omega_{i-1}}^{\prime}\right]_{\beta}\right)$. Due to Definition 4, it holds:

- $\omega_{i}=\omega_{i-1} t_{i} x_{i}$

- $M_{\omega_{i}}:=\left(M_{\omega_{i-1}} \backslash \bullet t_{i}\right) \cup t_{i} \bullet$ (i.e. $\left.t_{i} \in \operatorname{En}\left(M_{\omega_{i-1}}\right)\right)$;

- $\operatorname{Eft}\left(t_{i}\right) \leq I_{\omega_{i-1}}^{\prime}\left(t_{i}\right) \leq \operatorname{Lft}\left(t_{i}\right)$ in $B_{\omega}$;

- $\forall t \in \operatorname{En}\left(M_{\omega_{i}}\right), I_{\omega_{i}}^{\prime}(t)-x_{i}=\left\{\begin{array}{cc}0, & \text { if } \uparrow \operatorname{enabled}\left(t, M_{\omega_{i-1}}, t_{i}\right) \\ I_{\omega_{i-1}}^{\prime}(t), & \text { otherwise }\end{array}\right.$
Then, $E f t\left(t_{i}\right) \leq\left[I_{\omega_{i-1}}^{\prime}(t)\right]_{\beta} \leq L f t\left(t_{i}\right)$, because $\beta$ is a solution of $B_{\omega}$. Therefore, $t_{i}$ can fire from the state $\left(M_{\omega_{i-1}},\left[I_{\omega_{i-1}}^{\prime}\right]_{\beta}\right)$ By Definition 2, we have that $\left(M_{0}, I_{0}\right) \stackrel{\left[\omega_{i-1}\right]_{\beta}}{\rightarrow}\left(M_{\omega_{i-1}},\left[I_{\omega_{i-1}}^{\prime}\right]_{\beta}\right) \stackrel{t_{i}}{\rightarrow}\left(M_{\omega_{i}},\left[I_{\omega_{i}}^{\prime}\right]_{\beta}-\beta\left(x_{i}\right)\right) \stackrel{\beta\left(x_{i}\right)}{\rightarrow}\left(M_{\omega_{i}},\left[I_{\omega_{i}}^{\prime}\right]_{\beta}\right)$. Hence, it is true that $\left(M_{0}, I_{0}\right) \stackrel{[\omega]_{\beta}}{\longrightarrow}\left(M_{\omega},\left[I_{\omega}^{\prime}\right]_{\beta}\right)$. Moreover, for all $1 \leq i \leq k$, we have $\left[I^{\prime} \omega_{i-1}\left(t_{i}\right)\right]_{\beta}=I_{i-1}^{\prime}\left(t_{i}\right)$.

\section{Proof of Proposition 1.}

Let $\omega=x_{0} t_{1} x_{1} \ldots t_{k} x_{k} ; \beta_{i}$ be the functions from Definition 5 , with $0 \leq i \leq k ; \beta_{\omega}=\beta_{k}$; and $\mathcal{B}_{\omega}$ be the set of the variable parts of the inequalities from $B_{\omega}$. In order to show that the assignment $\beta_{\omega}$ is a solution of $\omega$, we consider an important property of the mappings $\beta_{i}$, for all $0 \leq i \leq k$.

Claim. For all $g \in \mathcal{B}_{\omega}$ and $0 \leq i \leq k$, it holds that $\left\lceil[g]_{\beta_{i}}\right\rceil \geq\left\lfloor[g]_{\beta_{0}}\right\rfloor$ and $\left[[g]_{\beta_{i}}\right\rfloor \leq\left\lceil[g]_{\beta_{0}}\right\rceil$.

Proof. We shall prove by induction on $i$.

$i=0$. Obvious.

$i>0$. Take an arbitrary $g \in \mathcal{B}_{\omega}$. By the induction hypothesis, we have that $\left[[g]_{\beta_{i-1}}\right] \geq\left\lfloor[g]_{\beta_{0}}\right\rfloor$ and $\left\lfloor[g]_{\beta_{i-1}}\right\rfloor \leq\left\lceil[g]_{\beta_{0}}\right]$. Let $\beta_{i}$ be the function from Definition 5 .

Assume that does not exist $\bar{h} \in \mathcal{B}_{\omega}$ s.t. $\left\lceil[h]_{\underline{\beta}_{i}}\right]<\left\lfloor[h]_{\beta_{0}}\right\rfloor$. Then, $[g]_{\beta_{i}}=[g]_{\underline{\beta_{i}}}$ and $[g]_{\beta_{i}} \leq$ $[g]_{\beta_{i-1}}$, due to Definition 5. Then $\left[[g]_{\beta_{i}}\right\rceil=\left\lceil[g]_{\beta_{i}}\right\rceil \geq\left\lfloor[g]_{\beta_{0}}\right\rfloor$ and $\left\lfloor[g]_{\beta_{i}}\right\rfloor \leq\left\lfloor[g]_{\beta_{i-1}}\right\rfloor \leq\left\lceil\lfloor g\rfloor_{\beta_{0}}\right\rceil$. Assume that there is exists $h \in \mathcal{B}_{\omega}$ s.t. $\left\lceil[h]_{\beta_{i}}\right]<\left[[h]_{\beta_{0}}\right\rfloor$. Then, $[g]_{\beta_{i}} \geq[g]_{\beta_{i-1}}$, by Definition 5 . Therefore, $\left\lceil[g]_{\beta_{i}}\right\rceil \geq\left\lceil[g]_{\beta_{i-1}}\right\rceil \geq\left[[g]_{\beta_{0}}\right\rfloor$. Suppose a contrary, i.e. $\left[[g]_{\beta_{i}}\right\rfloor>\left\lceil[g]_{\beta_{0}}\right]$. Then, $[g]_{\beta_{i}} \geq$ $\left\lfloor[g]_{\beta_{i}}\right\rfloor \geq\left[[g]_{\beta_{0}}\right\rceil+1$. According to Definition 5, $x_{k-i}$ appears in $g$ and $h$ and, moreover, $[h]_{\beta_{i}}=$ $[h]_{\beta_{i-1}}-\beta_{i-1}\left(x_{k-i}\right)+\beta_{i}\left(x_{k-i}\right)=[h]_{\beta_{i-1}}-\beta_{i-1}\left(x_{k-i}\right)+\left[\beta_{i-1}\left(x_{k-i}\right)\right] \leq[h]_{\beta_{i-1}}-$

$\beta_{i-1}\left(x_{k-i}\right)+\left\lfloor\beta_{i-1}\left(x_{k-i}\right)\right\rfloor+1=[h]_{\underline{\beta_{i}}}+1$. As $[h]_{\underline{\beta_{i}}}+1 \leq\left\lceil[h]_{\beta_{i}}\right\rceil+1 \leq\left\lfloor[h]_{\beta_{0}}\right\rfloor$, we have $[h]_{\beta_{i}} \leq \mid[h]_{\beta_{0}}$. Let $S: \mathbb{N} \times \mathbb{N} \rightarrow \mathbb{R}_{\geq 0}$ be such that $S(\bar{a}, b)=\sum_{j=a}^{b} x_{j}$, if $a<b$, and $S(a, b)=$ 0 , otherwise. Due to Definition 4 , it holds that $h=S(m, n)$ and $g=S\left(m^{\prime}, n^{\prime}\right)$, where $0 \leq$ $m, m^{\prime} \leq k-i \leq n, n^{\prime} \leq k$. By the construction of $\beta_{k}$, we have that $[S(a, b)]_{\beta_{c}}=[S(a, b)]_{\beta_{0}}$, if $0 \leq b<k-c \leq k$; and $[S(a, b)]_{\beta_{c}}=[S(a, b)]_{\beta_{k}}$, if $0 \leq k-c \leq a \leq k$. Therefore, we obtain the following:

(1) $[S(k-i, n)]_{\beta_{i}} \leq\left\lfloor[S(k-i, n)]_{\beta_{0}}\right\rfloor$, due to $[S(m, k-i-1)]_{\beta_{i}}=[S(m, k-i-$

1) $]_{\beta_{0}}$ and $[h]_{\beta_{i}} \leq\left\lceil[h]_{\beta_{i}}\right\rceil<\left\lfloor[h]_{\beta_{0}}\right\rfloor$

(2) $\left[S\left(k-i, n^{\prime}\right)\right]_{\beta_{i}} \geq\left[\left[S\left(k-i, n^{\prime}\right)\right]_{\beta_{0}}\right]+1$, due to $\left[S\left(m^{\prime}, k-i-1\right)\right]_{\beta_{i}}=\left[S\left(m^{\prime}, k-i-\right.\right.$

1) $]_{\beta_{0}}$ and $[g]_{\beta_{i}} \geq\left[[g]_{\beta_{0}}\right\rceil+1$

(3) $\left[S\left(m^{\prime}, n\right)\right]_{\beta_{k-n-1}}=\left[S\left(m^{\prime}, n\right)\right]_{\beta_{0}}$;

(4) $\left[S\left(m, n^{\prime}\right)\right]_{\beta_{k-n \prime-1}}=\left[S\left(m, n^{\prime}\right)\right]_{\beta_{0}}$;

(5) $\left[S\left(n+1, n^{\prime}\right)\right]_{\beta_{k-n-1}}=\left[S\left(n+1, n^{\prime}\right)\right]_{\beta_{i}}=\left[S\left(n+1, n^{\prime}\right)\right]_{\beta_{k}}$;

(6) $\left[S\left(n^{\prime}+1, n\right)\right]_{\beta_{k-n^{\prime}-1}}=\left[S\left(n^{\prime}+1, n\right)\right]_{\beta_{i}}=\left[S\left(n^{\prime}+1, n\right)\right]_{\beta_{k}}$.

Three cases are admissible:

- $n=n^{\prime}$. Then, (1) contradicts (2).

$-n<n^{\prime}$. Then, it holds that $[g]_{\beta_{k-n-1}}=\left[S\left(m^{\prime}, n\right)\right]_{\beta_{k-n-1}}+\left[S\left(n+1, n^{\prime}\right)\right]_{\beta_{k-n-1}}={ }^{(3)}$ $\left[S\left(m^{\prime}, k-i-1\right)\right]_{\beta_{0}}+[S(k-i, n)]_{\beta_{0}}+\left[S\left(n+1, n^{\prime}\right)\right]_{\beta_{k-n-1}} \geq \geq^{(1),(5)}\left[S\left(m^{\prime}, k-i-\right.\right.$

1) $]_{\beta_{0}}+[S(k-i, n)]_{\beta_{i}}+\left[S\left(n+1, n^{\prime}\right)\right]_{\beta_{i}} \geq^{(2)}\left[[g]_{\beta_{0}}\right]+1$. This contradicts the induction hypothesis, because $k-n \leq i$. 
$-n>n^{\prime}$. Then, it holds that $[h]_{\beta_{k-n^{\prime}-1}}=\left[S\left(m, n^{\prime}\right)\right]_{\beta_{k-n^{\prime}-1}}+\left[S\left(n^{\prime}+\right.\right.$ $1, n)]_{\beta_{k-n^{\prime}-1}}{ }^{(4)}[S(m, k-i-1)]_{\beta_{0}}+\left[S\left(k-i, n^{\prime}\right)\right]_{\beta_{0}}+\left[S\left(n^{\prime}+1, n\right)\right]_{\beta_{k-n^{\prime}-1}} \leq^{(2),(6)}$ $[S(m, k-i-1)]_{\beta_{0}}+\left[S\left(k-i, n^{\prime}\right)\right]_{\beta_{i}}-1+\left[S\left(n^{\prime}+1, n\right)\right]_{\beta_{i}} \leq^{(1)}\left\lfloor[h]_{\beta_{0}}\right\rfloor-1$. We get a contradiction the induction hypothesis, because $k-n^{\prime} \leq i$.

By Definition $4, x_{k}$ does not appear in $B_{\omega}$. Then, $\beta_{0}$ is a solution of $B_{\omega}$, according to Definition 5 . Take an arbitrary $g$ from $\mathcal{B}_{\omega}$. Then, it holds that $(a \leq g \leq b) \in B_{\omega}$, where $a, b \in \mathbb{Z}$. By Claim, we have $\left[[g]_{\beta_{0}}\right\rfloor \leq[g]_{\beta_{\omega}} \leq\left\lceil[g]_{\beta_{0}}\right\rceil$. Due to $\beta_{0}$ being a solution, we obtain $a \leq[g]_{\beta_{0}} \leq b$ and, moreover $a \leq\left\lfloor[g]_{\beta_{0}}\right\rfloor,\left\lceil[g]_{\beta_{0}}\right\rfloor \leq b$, because $a, b \in \mathbb{N} \cup \infty$. Thus, it holds that $a \leq\left\lfloor[g]_{\beta_{0}}\right\rfloor \leq$ $[g]_{\beta_{\omega}} \leq\left\lceil[g]_{\beta_{0}}\right\rceil \leq b$. Therefore, $\beta_{\omega}$ is a solution of $B_{\omega}$

Proof of Lemma 2

a) Take an arbitrary $1 \leq k \leq n$. By definitions, we have that $\operatorname{Cut}\left(E_{k}\right)=\left(\bullet T N \cup E_{k} \bullet\right) \backslash \cdot E_{k}=$ $\left(\bullet T N \cup E_{k-1} \bullet \cup e_{k} \bullet\right) \backslash\left(\bullet E_{k-1} \cup \bullet e_{k}\right)$. As $T N$ is an acyclic net, we obtain that $\bullet e_{k} \cap e_{k} \bullet=\varnothing$ and $\bullet E_{k-1} \cap e_{k} \bullet=\varnothing$. Then, it holds that $\operatorname{Cut}\left(E_{k}\right)=\left(\left(\left(\bullet N \cup E_{k-1} \bullet\right) \backslash \bullet E_{k-1}\right) \backslash \bullet e_{k}\right) \cup e_{k} \bullet=$ $\left(\operatorname{Cut}\left(E_{k-1}\right) \backslash \bullet e_{k}\right) \cup e_{k} \bullet$

b) Take arbitrary $1 \leq k \leq n$. The case with $\bullet e_{k}=\emptyset$ is trivial. Suppose $b \in \bullet e_{k}$. By definition, we have that $\operatorname{Cut}\left(E_{k-1}\right)=\left(\bullet T N \cup E_{k-1} \bullet\right) \backslash \bullet E_{k-1}$. Due to $T N$ being a causal net, $b \notin \bullet e_{i}$, for all $1 \leq i<k$. Hence, $b \notin \bullet E_{k-1}$. If $b \in \bullet T N$, then $b \in \operatorname{Cut}\left(E_{k-1}\right)$. Consider the case when $b \notin$ -TN. Then, there is $e_{i}$ such that $b \in e_{i} \bullet$. Clearly, $e_{i} \prec e_{k}$. This implies that $i<k$, in the linearization $\rho$. Hence, $b \in E_{k-1} \bullet$ and $b \in \operatorname{Cut}\left(E_{k-1}\right)$

c) As $\operatorname{Cut}\left(E_{0}\right)=\cdot T N=\{b \in B \mid \cdot b=\emptyset\}$, we have $\operatorname{Cut}\left(E_{0}\right)$ is a co-set. Suppose a contrary, i.e. there are $b, b^{\prime} \in \operatorname{Cut}\left(E_{k}\right)$, for some $1 \leq k \leq n$, such that $b \prec b^{\prime}$. As $\rho$ is a linearization, we have $b G e_{i} \ldots e_{j} G b^{\prime}$, with $i \leq j$. Due to $\operatorname{Cut}\left(E_{k}\right)=\left(\bullet T N \cup E_{k} \bullet\right) \backslash \bullet E_{k}$, we get $b, b^{\prime} \notin \bullet E_{k}$ and $b^{\prime} \in E_{k} \bullet$. Since $\bullet b^{\prime}=e_{j}$, it holds that $j \leq k$, i.e. $i \leq k$. This means that $b \in \bullet e_{i} \subseteq \bullet E_{k}$, contradicting $b \notin \bullet E_{k}$. Thus, $\neg\left(b \prec b^{\prime}\right)$.

We shall show that $\operatorname{Cut}\left(E_{k}\right)$ is a cut, for all $0 \leq k \leq n$. Suppose a contrary, i.e. there exists $b \notin$ $\operatorname{Cut}\left(E_{k}\right)$, for some $0 \leq k \leq n$, such that $\neg\left(b \prec b^{\prime}\right)$ and $\neg\left(b^{\prime} \prec b\right)$, for all $b^{\prime} \in \operatorname{Cut}\left(E_{k}\right)$. W.l.o.g. assume $b \in \operatorname{Cut}\left(E_{i}\right)$, for some $0 \leq i \neq k \leq n$. Thanks to item a), $\operatorname{Cut}\left(E_{j}\right)=$ $\left(\operatorname{Cut}\left(E_{j-1}\right) \backslash \bullet e_{j}\right) \cup e_{j} \bullet$, for all $1 \leq j \leq n$. If $i<k$, then we get that $b G e_{l} \ldots e_{m} G b^{\prime}$, for some $b^{\prime} \in \operatorname{Cut}\left(E_{k}\right)$ and $i<l \leq m \leq k$, i.e. $b \prec b^{\prime}$, because $T N$ is a causal net. If $i>k$, then we have that $b^{\prime} G e_{l} \ldots e_{m} G b$, for some $b^{\prime} \in \operatorname{Cut}\left(E_{k}\right)$ and $k<l \leq m \leq i$, i.e. $b^{\prime}<b$, again because $T N$ is a causal net. Thus, $\operatorname{Cut}\left(E_{k}\right)$ is a cut, for all $0 \leq k \leq n$.

Proof of Lemma 3.

a), b) We shall verify the items by induction on $0 \leq i \leq n$.

$i=0$. By definitions, it holds that $\operatorname{Cut}\left(E_{0}\right)=\bullet T N$.

a) The restriction of $\varphi$ to $\operatorname{Cut}\left(E_{0}\right)$ is a bijection between $\operatorname{Cut}\left(E_{0}\right)$ and $M_{0}$, due to Definition 7.

b) As Age $(b)=0$, for all $b \in \cdot T N$, $\operatorname{Clock}\left(\operatorname{Cut}\left(E_{0}\right), t\right)=0=I_{0}(t)$, for all $t \in \operatorname{En}\left(M_{0}\right)$, thanks to $\mathcal{T} \mathcal{N}$ being $T$-restricted.

$i>0$. By the induction hypothesis, the items hold for $i-1$. We now check them for $i$. Two cases are admissible.
Case 1: $\varphi\left(e_{i}\right)=\sqrt{ }$. Then, it holds that $M_{i-1}=M_{i}$, by Definition 2. According to Definition 7 , we have that $\varphi\left(e_{i} \bullet\right)=\varphi\left(\bullet e_{i}\right)$ and $\bullet e_{i}, e_{i} \bullet$ are cuts, i.e. $\bullet e_{i} \neq \varnothing, e_{i} \bullet \neq \varnothing$. In addition, we have that $\bullet e_{i} \subseteq \operatorname{Cut}\left(E_{i-1}\right), e_{i} \bullet \subseteq \operatorname{Cut}\left(E_{i}\right)$, and $\operatorname{Cut}\left(E_{i-1}\right), \operatorname{Cut}\left(E_{i}\right)$ are cuts, due to Lemma 2. Hence, we get that $\bullet e_{i}=\operatorname{Cut}\left(E_{i-1}\right)$ and $\operatorname{Cut}\left(E_{i}\right)=e_{i} \bullet$, and, moreover, $\varphi\left(\operatorname{Cut}\left(E_{i}\right)\right)=\varphi\left(\operatorname{Cut}\left(E_{i-1}\right)\right)=M_{i-1}=M_{i}$.

a) As the restriction of $\varphi$ to $e_{i} \bullet$ is an injection, by Definition 7, the restriction of $\varphi$ to $\operatorname{Cut}\left(E_{i}\right)$ is a bijection between $\operatorname{Cut}\left(E_{i}\right)$ and $M_{i}$.

b) Take an arbitrary $t \in E n\left(M_{i}\right)$. According to Definition 2, we have that $t \in E n\left(M_{i-1}\right)$ and $I_{i-1}(t)+\varphi\left(e_{i}\right)=I_{i}(t)$. By definition, Age $(b)=A g e\left(b^{\prime}\right)+\varphi\left(e_{i}\right)$, with $b^{\prime} \in \operatorname{Cut}\left(E_{i-1}\right)$ and $\varphi(b)=\varphi\left(b^{\prime}\right)$, for all $b \in e_{i} \bullet \in \operatorname{Cut}\left(E_{i}\right)$. Then, due to $\mathcal{T} \mathcal{N}$ being $T$-restricted, we obtain that $\operatorname{Clock}\left(\operatorname{Cut}\left(E_{i}\right), t\right)=\min (\{A g e(b) \mid \varphi(b) \in \bullet t$ $\left.\left.b \in \operatorname{Cut}\left(E_{i}\right)\right\}\right)=\min \left(\left\{\operatorname{Age}(b)+\varphi\left(e_{i}\right) \mid \varphi(b) \in \bullet t, b \in \operatorname{Cut}\left(E_{i-1}\right)\right\}\right)=$ $\operatorname{Clock}\left(\operatorname{Cut}\left(E_{i-1}\right), t\right)+\varphi\left(e_{i}\right)=I_{i-1}(t)+\varphi\left(e_{i}\right)=I_{i}(t)$.

Case 2: $\varphi\left(e_{i}\right) \in T$. Then, $M_{i}=\left(M_{i-1} \backslash \bullet \varphi\left(e_{i}\right)\right) \cup \varphi\left(e_{i}\right) \bullet$, according to Definition 2 . Due to Definition 7, the restrictions of $\varphi$ to $\bullet e_{i}\left(e_{i} \bullet\right)$ are bijections between $\bullet e_{i}\left(e_{i} \bullet\right)$ and $\bullet \varphi\left(e_{i}\right)\left(\varphi\left(e_{i}\right) \bullet\right)$.

a) By the inductive hypothesis, the restriction of $\varphi$ to $\operatorname{Cut}\left(E_{i-1}\right)$ is a bijection between $\operatorname{Cut}\left(E_{i-1}\right)$ and $M_{i-1}$. Then, due to Definition 2, we get that $M_{i}=\left(\varphi\left(\operatorname{Cut}\left(E_{i-1}\right)\right) \backslash\right.$ - $\left.\varphi\left(e_{i}\right)\right) \cup \varphi\left(e_{i}\right) \bullet=\varphi\left(\left(\operatorname{Cut}\left(E_{i-1}\right) \backslash \bullet e_{i}\right) \cup e_{i} \bullet\right)=\varphi\left(\operatorname{Cut}\left(E_{i}\right)\right)$, using Lemma 2(a). Since $\mathcal{T} \mathcal{N}$ is contact-free, we obtain that $\varphi\left(\operatorname{Cut}\left(E_{i-1}\right) \backslash \bullet e_{i}\right) \cap$ $\varphi\left(e_{i} \bullet\right)=\varnothing$. Therefore, the restriction of $\varphi$ to $\operatorname{Cut}\left(E_{i}\right)$ is a bijection between $\operatorname{Cut}\left(E_{i}\right)$ and $M_{i}$.

b) Take an arbitrary $t \in E n\left(M_{i}\right)$. Assume that $\uparrow$ enabled $\left(t, M_{i-1}, \varphi\left(e_{i}\right)\right)$ is true. Then we have that $t \notin E n\left(M_{i-1} l \bullet \varphi\left(e_{i}\right)\right)$ or $t=\varphi\left(e_{i}\right)$, by Definition 2. If $t=\varphi\left(e_{i}\right)$ then $t \in E n\left(M_{i-1}\right)$ and $t \notin E n\left(M_{i-1} \backslash \bullet t\right)=E n\left(M_{i-1} \backslash \bullet \varphi\left(e_{i}\right)\right)$. So, $t \notin E n\left(M_{i-1} \backslash\right.$ - $\left.\varphi\left(e_{i}\right)\right)$. Due to Definition 2, it is true that $\operatorname{En}\left(M_{i}\right)=\operatorname{En}\left(\left(M_{i-1} \backslash \bullet \varphi\left(e_{i}\right)\right) \cup \varphi\left(e_{i} \bullet\right)\right)$. Thanks to $\mathcal{T} \mathcal{N}$ is $T$-restricted, we get $\bullet t \neq \emptyset$ and $\varphi\left(e_{i} \bullet\right) \neq \emptyset$. Since $t \in \operatorname{En}\left(M_{i}\right)$ and $t \notin E n\left(M_{i-1} \backslash \bullet \varphi\left(e_{i}\right)\right)$, we have that $\bullet t \cap \varphi\left(e_{i} \bullet\right) \neq \emptyset$. According to Lemma 2(a), it holds that $e_{i} \bullet \subseteq \operatorname{Cut}\left(E_{i}\right)$. Hence, there is $b \in \operatorname{Cut}\left(E_{i}\right)$ such that $\varphi(b) \in \bullet t$ and $\operatorname{Age}(b)=0$. Therefore, due to $\mathcal{T N}$ being $T$-restricted, it is true that $\operatorname{Clock}\left(\operatorname{Cut}\left(E_{i}\right), t\right)=\left(\min \left\{\operatorname{Age}(b) \mid \varphi(b) \in \bullet t, b \in \operatorname{Cut}\left(E_{i}\right)\right\}\right)=0$.

Thus, $\operatorname{Clock}\left(\operatorname{Cut}\left(E_{i}\right), t\right)=I_{i}(t)$, due to Definition 2.

Suppose that $\uparrow$ enabled $\left(t, M_{i-1}, \varphi\left(e_{i}\right)\right)$ is false. Then, we get that $t \in \operatorname{En}\left(M_{i-1}\right)$ $\left.\bullet \varphi\left(e_{i}\right)\right)$ and $t \neq \varphi\left(e_{i}\right)$, by Definition 2 . Hence, $\bullet \varphi\left(e_{i}\right) \cap \bullet t=\emptyset$, i.e. $\varphi\left(\bullet e_{i}\right) \cap \bullet t=$ $\emptyset$. As $\mathcal{T} \mathcal{N}$ is contact-free, it holds that $\left(M_{i-1} \backslash \bullet \varphi\left(e_{i}\right)\right) \cap \varphi\left(e_{i}\right) \bullet=\varnothing$. This means that, $\varphi\left(e_{i}\right) \bullet \cap \bullet t=\emptyset$, i.e. $\varphi\left(e_{i} \bullet\right) \cap \bullet t=\emptyset$. Therefore, if $\varphi(b) \in \bullet t$, then $b \notin e_{i} \bullet$ and $b \notin \bullet e_{i}$. By the induction hypothesis, we have that $I_{i-1}(t)=$ $\operatorname{Clock}\left(\operatorname{Cut}\left(E_{i-1}\right), t\right)=\min \left(\left\{\operatorname{Age}(b) \mid \varphi(b) \in \bullet t, b \in \operatorname{Cut}\left(E_{i-1}\right)\right\}\right)=$

$\min \left(\left\{\operatorname{Age}(b) \mid \varphi(b) \in \bullet t, b \in\left(\operatorname{Cut}\left(E_{i-1}\right) \backslash \bullet e_{i}\right) \cup e_{i} \bullet\right\}\right)$. Thanks to Lemma 2(a), we obtain that $I_{i-1}(t)=\operatorname{Clock}\left(\operatorname{Cut}\left(E_{i}\right), t\right)$. Thus, it holds that $I_{i}(t)=I_{i-1}(t)=$ $\operatorname{Clock}\left(\operatorname{Cut}\left(E_{i}\right), t\right)$, due to Definition 2 .

c) Assume that $i<n$ and $\varphi\left(e_{i+1}\right) \in \operatorname{En}\left(M_{i}\right)$. Then, $\varphi\left(e_{i+1}\right) \in \operatorname{En}\left(\varphi\left(\operatorname{Cut}\left(E_{i}\right)\right)\right)$, due to item a). By definition, due to $\mathcal{T} \mathcal{N}$ being $T$-restricted, we have that $\operatorname{Clock}\left(\operatorname{Cut}\left(E_{i}\right), \varphi\left(e_{i+1}\right)\right)=$ $\min \left(\left\{\operatorname{Age}(b) \mid \varphi(b) \in \bullet \varphi\left(e_{i+1}\right), b \in \operatorname{Cut}\left(E_{i}\right)\right\}\right)$. Take an arbitrary $b \in \operatorname{Cut}\left(E_{i}\right)$ such that $\varphi(b) \in \bullet \varphi\left(e_{i+1}\right)$. Thanks to the definition of a homomorphism, it holds that $\varphi(b) \in$ $\varphi\left(\bullet e_{i+1}\right)$. Hence, $b \in \bullet e_{i+1}$, due to item a). By virtue of Lemma $2(\mathrm{~b}), \bullet e_{i+1} \subseteq \operatorname{Cut}\left(E_{i}\right)$. This implies that $\operatorname{Clock}\left(\operatorname{Cut}\left(E_{i}\right), \varphi\left(e_{i+1}\right)\right)=\operatorname{Clock}\left(\bullet \bullet_{i+1}, \varphi\left(e_{i+1}\right)\right)$. Therefore, $\operatorname{Clock}\left(\bullet e_{i+1}, \varphi\left(e_{i+1}\right)\right)=I_{i}\left(\varphi\left(e_{i+1}\right)\right)$, due to item (b). 
a) By the construction of $T N^{*}$, we have the following. First, $B$ and $E$ are finite sets. Second, $G \subseteq$ $(B \times E) \cup(E \times B)$ is a flow relation such that $e_{j} G_{i} b_{j p} G_{i} e_{i}$, i.e. $j<i$. Hence, $T N^{*}$ is acyclic. Third, $l^{*}: E \rightarrow$ Act $\cup\{t i c k\}$ is a labeling function. By the construction of $G$, we obtain that $\cdot b_{0, p}=\emptyset$ and $\bullet b_{i, p}=\left\{e_{i}\right\}$, for all $1 \leq i \leq n$. Therefore, $\left|\cdot b_{i, p}\right| \leq 1$, for all $0 \leq i \leq n$. Suppose a contrary, i.e. $\left|b_{j, p} \bullet\right|>1$, for some $b_{j, p} \in B$. Then, there exists $i \neq i^{\prime}$ such that $\left\{b_{j, p}\right\} \in$ $\cdot e_{i}$ and $\left\{b_{j, p}\right\} \in \bullet e_{i^{\prime}}$. Hence, by the construction of $G$, we get that $j<i, b_{j, p} \in C_{i-1}$ and $j<i^{\prime}$, $b_{j, p} \in C_{i^{\prime}-1}$. W.1.o.g. assume $i<i^{\prime}$. As $C_{l}=\left(C_{l-1} \backslash \bullet e_{l}\right) \cup e_{l} \bullet$, for all $1 \leq l \leq n$, there exists $i \leq$ $k \leq i^{\prime}-1$ such that $b_{j, p} \in e_{k} \bullet$. According to the construction of $G_{k}, j=k$, contradicting $j<$

b) Due to Definition 9 , every event of $T N^{*}$ appears in the sequence $\rho^{*}=e_{1} \ldots e_{n}$ exactly once. By the construction of $G$, it holds that $e_{i}<e_{j}$ implies $i<j$.

c) As $C_{0}=\bullet T N^{*}$, we get $C_{0}=\operatorname{Cut}\left(E_{0}\right)$. Thanks to items a), b) and Lemma 2(a), we obtain $C_{i}=$ $\operatorname{Cut}\left(E_{i}\right)$, for $0 \leq i \leq n$.

\section{Proof of Lemma 5}

Claim. The restriction of $\varphi^{*}$ to $C_{i}$ is a bijection between $C_{i}$ and $M_{i}$, for all $0 \leq i \leq n$.

Proof. We prove by induction on $0 \leq i \leq n$.

$i=0$. Then, $C_{0}=B_{0}=\left\{b_{0, p} \mid p \in M_{0}\right\}$, i.e. the restriction of $\varphi^{*}$ to $C_{0}$ is a bijection between $C_{0}$ and $M_{0}$.

$i>0$. By the induction hypothesis, the restriction of $\varphi^{*}$ to $C_{i-1}$ is a bijection between $C_{i-1}$ and $M_{i-1}$. Two cases are admissible.

Let $\varphi^{*}\left(e_{i}\right)=\overline{t_{i}}=\sqrt{ }$. By Definition 2, we have $M_{i}=M_{i-1}$. Thanks to the construction of $G_{i}$ and $C_{i}$, it holds that $C_{i}=\left(C_{i-1} \backslash \bullet e_{i}\right) \cup e_{i} \bullet=e_{i} \bullet=C_{i-1}$. Then, the restriction of $\varphi^{*}$ to $C_{i}$ is a bijection between $C_{i}$ and $M_{i}$

Let $\varphi^{*}\left(e_{i}\right)=\overline{t_{i}} \in T$. By Definition 2, we have that $M_{i}=\left(M_{i-1} \backslash \bullet \varphi^{*}\left(e_{i}\right)\right) \cup \varphi^{*}\left(e_{i}\right) \bullet$. Take an arbitrary $p \in \bullet \varphi^{*}\left(e_{i}\right)$ (it exists because $\mathcal{T N}$ is $T$-restricted). Then, $p \in M_{i-1}$ and, moreover, there exists $b_{j, p} \in C_{i-1}$, due to the induction hypothesis. Thanks to the construction of $G_{i}$, we get that $\bullet e_{i}=\left\{b_{j, p} \mid p \in \bullet \varphi^{*}\left(e_{i}\right) \wedge b_{j, p} \in C_{i-1}\right\}$ and $e_{i} \bullet=\left\{b_{i, p} \mid p \in \varphi^{*}\left(e_{i}\right) \bullet\right\}$. Hence, the restriction of $\varphi^{*}$ to $\bullet e_{i}\left(e_{i} \bullet\right)$ is a bijection between $\bullet e_{i}\left(e_{i} \bullet\right)$ and $\bullet \varphi^{*}\left(e_{i}\right)\left(\varphi^{*}\left(e_{i}\right) \bullet\right)$. Then, $\varphi^{*}\left(C_{i}\right)=$ $\varphi^{*}\left(\left(C_{i-1} \backslash \bullet e_{i}\right) \cup e_{i} \bullet\right)=\left(\varphi^{*}\left(C_{i-1}\right) \backslash \varphi^{*}\left(\bullet e_{i}\right)\right) \cup \varphi^{*}\left(e_{i} \bullet\right)=\left(M_{i-1} \backslash \bullet \varphi^{*}\left(e_{i}\right)\right) \cup \varphi^{*}\left(e_{i}\right) \bullet=M_{i}$. Due to $\mathcal{T \mathcal { N }}$ being contact-free, we obtain $\left(M_{i-1} \backslash \bullet \varphi^{*}\left(e_{i}\right)\right) \cup \varphi^{*}\left(e_{i}\right) \bullet=\emptyset$.

Therefore, the restriction of $\varphi^{*}$ to $C_{i}$ is a bijection between $C_{i}$ and $M_{i}$.

By definition, we have that $\varphi^{*}(B) \subseteq P, \varphi^{*}(E) \subseteq(T \cup\{\sqrt{ }\})$, and $l^{*}(e)=\left\{\begin{array}{c}\text { tick, if } \varphi^{*}(e)=\sqrt{ } \text {, } \\ L\left(\varphi^{*}(e)\right) \text {, otherwise }\end{array}\right.$, for all $e \in E$.

Take an arbitrary $1 \leq i \leq n$

Assume $\varphi^{*}\left(e_{i}\right) \in T$. Due to Claim, the restriction of $\varphi^{*}$ to $\bullet e_{i}\left(e_{i} \bullet\right)$ is a bijection between $\bullet e_{i}\left(e_{i} \bullet\right)$ and $\bullet \varphi^{*}\left(e_{i}\right)\left(\varphi^{*}\left(e_{i}\right) \bullet\right)$.

Assume $\varphi^{*}\left(e_{i}\right)=\sqrt{ }$. Thanks to the construction of $G_{i}$ and $C_{i}$, we have that $C_{i-1}=\bullet e_{i}=e_{i} \bullet=C_{i}$. By Claim, the restriction of $\varphi$ to $\bullet e_{i}$ and the restriction of $\varphi$ to $e_{i} \bullet$ are injections. As $\mathcal{T N}$ is $T$ restricted, we obtain $\bullet e_{i} \neq \varnothing$ and $e_{i} \bullet \neq \varnothing$. According to Lemma 4(c), we have that $\bullet e_{i}=\operatorname{Cut}\left(E_{i-1}\right)$ and $e_{i} \bullet=\operatorname{Cut}\left(E_{i}\right)$. Due to Lemma 2(c), $\bullet e_{i}$ and $e_{i} \bullet$ are cuts.

As $\bullet T N^{*}=C_{0}$, by construction, the restriction of $\varphi^{*}$ to $\bullet T N^{*}$ is a bijection between $\bullet T N^{*}$ and $M_{0}$, due to Claim.

Thus, $\varphi^{*}$ is a homomorphism from $T N^{*}$ to $\mathcal{T} \mathcal{N}$, by virtue of Lemma 4(a).

\section{Information about authors / Информация об авторах}

Alexey Yurievich ZUBAREV, PhD student. Research interests: parallel computing, Petri nets.

Алексей Юрьевич ЗУБАРЕВ, аспирант. Научные интересы: параллельные вычисления, сети Петри.

Irina Bonaventurovna VIRBITSKAITE - Doctor of Physical and Mathematical Sciences, Professor, Head of the Laboratory of the Theory of Parallel Processes at IIS SB RAS, Professor at NSU. Research interests: theory of parallel processes; specification and verification of parallel real-time systems.

Ирина Бонавентуровна ВИРБИЦКАЙТЕ - доктор физико-математических наук, профессор, заведующая лабораторией теории параллельных процессов в ИСИ СО РАН, профессор НГУ. Научные интересы: теория параллельных процессов; спецификация и верификация параллельных систем реального времени. 